\title{
INTERSECTION THEORY OF MODULI SPACE OF STABLE $N$-POINTED CURVES OF GENUS ZERO
}

\author{
SEAN KEEL
}

\begin{abstract}
We give a new construction of the moduli space via a composition of smooth codimension two blowups and use our construction to determine the Chow ring.
\end{abstract}

\section{INTRODUCTION}

This paper concerns the intersection theory of the moduli space of $n$-pointed stable curves of genus 0 ( $n$-pointed stable curves will be defined shortly). In [Kn] Knudsen constructs the space, which we call $\mathbf{X}_{n}$, and shows it is a smooth complete variety. We give an alternative construction of $\mathbf{X}_{n}$, via a sequence of blowups of smooth varieties along smooth codimension two subvarieties, and using our construction:

(1) We show that the canonical map from the Chow groups to homology (in characteristic zero)

$$
\mathbf{A}_{*}\left(\mathbf{X}_{n}\right) \stackrel{\mathrm{cl}}{\longrightarrow} H_{*}\left(\mathbf{X}_{n}\right)
$$

is an isomorphism.

(2) We give a recursive formula for the Betti numbers of $\mathbf{x}_{n}$.

(3) We give an inductive recipe for determining dual bases in the Chow ring $\mathbf{A}^{*}\left(\mathbf{X}_{n}\right)$.

(4) We calculate the Chow ring. It is generated by divisors, and we express it as a quotient of a polynomial ring by giving generators for the ideal of relations.

Once we have described $\mathbf{X}_{n}$ via blowups, our results follow from application of some general results on the Chow rings of regular blowups which we develop in an appendix.

We now sketch Knudsen's construction of $\mathbf{X}_{n}$, and then discuss our alternative method. Finally, we state explicitly the results on the intersection theory announced at the outset.

Fix an algebraically closed field $k$, (of arbitrary characteristic) over which all schemes discussed are assumed to be defined. Let $\mathbf{M}_{n}$ be the contravariant

Received by the editors August 1, 1989 and, in revised form, January 2, 1990.

1980 Mathematics Subject Classification (1985 Revision). Primary 14C17.

During this research the author was supported by a Sloan foundation doctoral dissertation fellowship. 
functor which sends a scheme $S$ to the collection of $n$-pointed curves of genus 0 over $S$ modulo isomorphisms.

Where by definition, a flat proper morphism $\mathscr{C} \stackrel{\pi}{\longrightarrow} S$ with $n$ distinct sections $s_{1}, s_{2}, \ldots, s_{n}$ is an $n$-pointed stable curve of genus 0 provided:

(1) The geometric fibers $\mathscr{C}_{s}$ of $\pi$ are reduced connected curves, with at worst ordinary double points, each irreducible component of which is isomorphic to $\mathbf{P}^{1}$.

(2) With $P_{i}=s_{i}(s), P_{i} \neq P_{j}$ for $i \neq j$.

(3) $P_{i}$ is a smooth point of $\mathscr{C}_{s}$.

(4) For each irreducible component of $\mathscr{C}_{s}$, the number of singular points of $\mathscr{C}_{s}$ which lie on it plus the number of $P_{i}$ on it is at least three.

(5) $\operatorname{dim} H^{1}\left(\mathscr{C}_{s}, \mathscr{O}_{\mathscr{C}_{s}}\right)=0$.

$\left((1)\right.$ and (5) imply that each $\mathscr{C}_{s}$ is a tree of $\left.\mathbf{P}^{1} \mathbf{s}\right)$

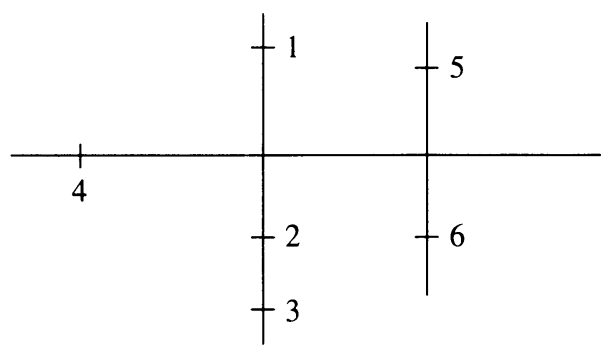

In the sequel, we will abbreviate the expression $n$-pointed stable curve of genus 0 by $n$-pointed curve, or simply curve if $n$ is clear from context. Two $n$-pointed curves $\mathscr{C} \stackrel{\pi}{\longrightarrow} S, s_{1}, s_{2}, \ldots, s_{n}$ and $\mathscr{C}^{\prime} \stackrel{\pi^{\prime}}{\longrightarrow} S, s_{1}^{\prime}, s_{2}^{\prime}, \ldots, s_{n}^{\prime}$ are isomorphic if there exists an isomorphism $f: \mathscr{C} \rightarrow \mathscr{C}^{\prime}$ over $S$ such that $f \circ s_{i}=s_{i}^{\prime}$.

Knudsen demonstrates that $\mathbf{M}_{n}$ is represented by a smooth complete variety $\mathbf{X}_{n}$ together with a universal curve $\mathbf{U}_{n} \stackrel{\pi}{\longrightarrow} \mathbf{X}_{n}$, and universal sections $\sigma_{1}, \sigma_{2}, \ldots, \sigma_{n}$.

In addition to representing $\mathbf{M}_{n}, \mathbf{X}_{n}$ gives an interesting compactification of the space of $n$ distinct points on $\mathbf{P}^{1}$ modulo automorphisms of $\mathbf{P}^{1}$ which is isomorphic to

$$
\underbrace{\mathbf{P}^{1} \backslash\{0,1, \infty\} \times \mathbf{P}^{1} \backslash\{0,1, \infty\} \times \cdots \times \mathbf{P}^{1} \backslash\{0,1, \infty\}}_{n-3 \text { factors }} \backslash \Delta
$$

since an automorphism of $\mathbf{P}^{1}$ is determined by its action on three distinct points. This space is contained in $\mathbf{X}_{n}$ as the open subset over which $\pi$ is smooth, hence the open set parameterizing $n$-pointed curves over $\operatorname{spec}(k)$ for which the curve is $\mathbf{P}^{1}$.

In this compactification, when two points come together, the limit is a curve with a new branch, containing the two points which came together.

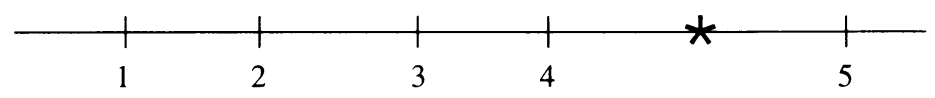

4 and 5 both approach the point labeled $*$ and the limit is 


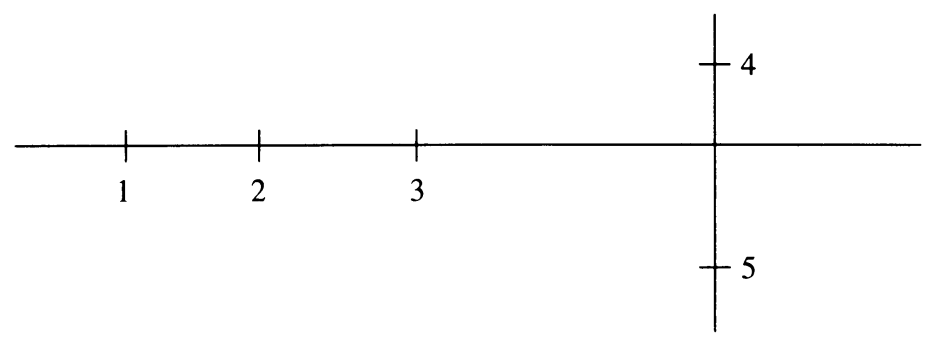

with the two branches meeting at $*$.

Knudsen's construction of $\mathbf{X}_{n}$ is inductive. He shows that the universal curve $\mathbf{U}_{n} \rightarrow \mathbf{X}_{n}$ is in fact $\mathbf{X}_{n+1}$, and that the universal curve over $\mathbf{X}_{n+1}$ can be constructed by blowing up $\mathbf{X}_{n+1} \times \mathbf{x}_{n} \mathbf{X}_{n+1}$ along a subscheme of the diagonal. His method relies on two functors, contraction and stabilization:

Given an $n+1$-pointed curve $\mathscr{C} \stackrel{\pi}{\longrightarrow} S$ with sections $s_{1}, s_{2}, \ldots, s_{n+1}$, an $n$ pointed curve $\mathscr{C}^{\prime} \stackrel{\pi}{\longrightarrow} S$ with sections $s_{1}^{\prime}, s_{2}^{\prime}, \ldots, s_{n}^{\prime}$ is a contraction of $\mathscr{C} \stackrel{\pi}{\longrightarrow}$ $S$ provided there is a commutative diagram

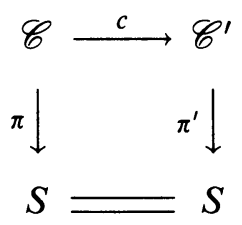

satisfying

(1) $c \circ s_{i}=s_{i}^{\prime}$ for $i \leq n$.

(2) Consider the induced morphism $c_{s}$ on a geometric fiber $\mathscr{C}_{s}$. Let $P=$ $s_{n+1}(s)$ and suppose $P$ lies on the irreducible component $E$. If the number of sections $s_{i}(s)$ other than $P$, plus the number of other components which $E$ meets, is at least three then $c_{s}$ is an isomorphism. Otherwise, $c_{s}$ contracts $E$ to a point, and the restriction of $c_{s}$ to $\mathscr{C}_{s} \backslash E$ is an isomorphism.

Thus if $\mathscr{C}_{s}$ with $P_{1}, P_{2}, \ldots, P_{n}$ is an $n$-pointed curve, then contraction leaves it alone. Otherwise, the component containing $P_{n+1}$ is contracted, and the resulting space together with $P_{1}, P_{2}, \ldots, P_{n}$ is an $n$-pointed curve.
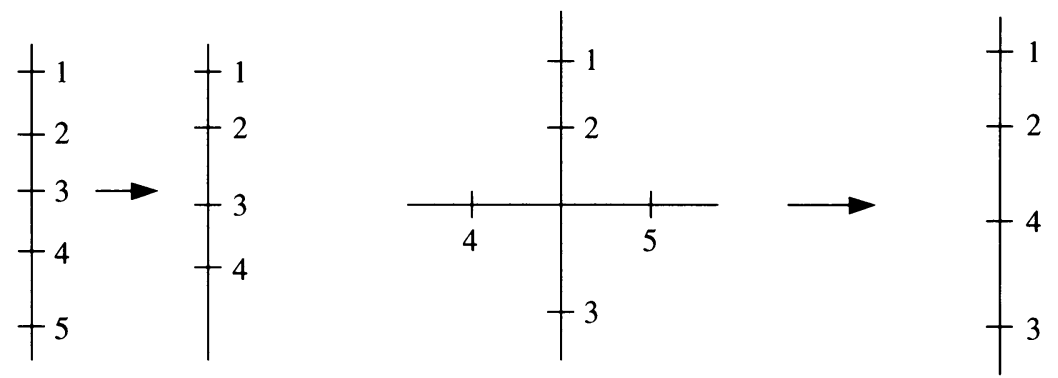

Knudsen shows that for any $n+1$-pointed curve, there exists up to unique isomorphism, exactly one contraction.

As for stabilization: Suppose $\mathscr{C} \stackrel{\pi}{\longrightarrow} S$ with $s_{1}, s_{2}, \ldots, s_{n}$ is an $n$-pointed curve with an additional section $s$. ( $s$ can be any section whatsoever.) Knudsen shows that there exists (up to a unique isomorphism) a unique $n+1$-pointed 
curve $\mathscr{C}^{s} \stackrel{\pi^{\prime}}{\longrightarrow} S$ with sections $s_{1}^{\prime}, \ldots, s_{n+1}^{\prime}$ such that $\mathscr{C}$ is the contraction of $\mathscr{C}^{s}$ along $s_{n+1}^{\prime}$ and such that $s_{n+1}^{\prime}$ is sent to the section $s . \mathscr{C}^{s}$ is obtained from $\mathscr{C}$ by a blowup which he describes explicitly. Knudsen also shows that contraction and stabilization commute with pullbacks.

The functorial upshot of these remarks is as follows: Suppose $\mathbf{U}_{n} \rightarrow \mathbf{X}_{n}$ with sections $\sigma^{1}, \ldots, \sigma^{n}$ represents $\mathbf{M}_{n}$ (i.e. $\mathbf{U}_{n} \rightarrow \mathbf{X}_{n}$ is the universal $n$-pointed curve). Then

$$
\mathbf{U}_{n} \times \mathbf{x}_{n} \mathbf{U}_{n} \rightarrow \mathbf{U}_{n}
$$

with the pulled back sections $\sigma^{1}, \ldots, \sigma^{n}$ and the additional section $\Delta$ (the diagonal map) is the universal $n$-pointed curve with an additional section and its stabilization

$$
\left(\mathbf{U}_{n} \times \mathbf{x}_{n} \mathbf{U}_{n}\right)^{s} \rightarrow \mathbf{U}_{n}
$$

is the universal $n+1$-pointed curve. In particular, $\mathbf{X}_{n+1}=\mathbf{U}_{n}$, and $\mathbf{U}_{n+1}$ is a blowup of $\mathbf{X}_{n+1} \times \mathbf{x}_{n} \mathbf{X}_{n+1}$.

Since $\mathbf{P}^{1} \rightarrow p t$ with sections $0,1, \infty$ represents $\mathbf{M}_{3}$ it follows that $\mathbf{X}_{3}=p t$, $\mathbf{X}_{4}=\mathbf{P}^{1}$ and $\mathbf{X}_{n+2}$ can be constructed inductively by blowing up $\mathbf{X}_{n+1} \times \mathbf{X}_{n} \mathbf{X}_{n+1}$.

The principal drawbacks, from our perspective, of this construction, are that $\mathbf{X}_{n+1} \times \mathbf{x}_{n} \mathbf{X}_{n+1}$ is not smooth, and the blowup is not along a regularly embedded subscheme. (Knudsen shows that the map $\mathbf{X}_{n+1} \stackrel{\pi}{\longleftarrow} \mathbf{X}_{n}$ looks locally on $\mathbf{X}_{n+1}$ and $\mathbf{X}_{n}$ like $U \times H \rightarrow U \times \mathbf{A}^{1}$, where $U$ is smooth, $H$ is the subvariety of $\mathbf{A}^{3}$ defined by $x y=t$, and the morphism $H \rightarrow \mathbf{A}^{1}$ sends $(x, y, t)$ to $t$. Thus locally $\mathbf{X}_{n+1} \times \mathbf{x}_{n} \mathbf{X}_{n+1}$ is the product $U \times$ (the affine cone $x y=z w$ ), and with this presentation $\Delta \hookrightarrow \mathbf{X}_{n+1} \times \mathbf{x}_{n} \mathbf{X}_{n+1}$ is locally the inclusion $U \times(x=z, y=$ $w) \hookrightarrow U \times(x y=z w)$.) We circumvent this obstacle by showing that $\pi$ can be factored as

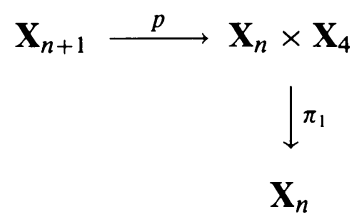

where $\pi_{1}$ is projection on the first factor, and $p$ is a composition of blowups of smooth varieties along smooth codimension two subvarieties.

In order to present this blowup description it is necessary to introduce various "vital" divisors on $\mathbf{X}_{n}$. For each subset $T \subset\{1,2, \ldots, n\}$ with $|T| \geq 2$ and $\left|T^{C}\right| \geq 2(|T|$ indicates the number of elements in $T)$ we let $D^{T} \hookrightarrow \mathbf{X}_{n}$ be the divisor whose generic element is a curve with two components, the points of $T$ on one branch, the points of $T^{C}$ on the other.

Observe that $D^{T}=D^{T^{C}}$. In order to eliminate this duplication it will occasionally be useful to assume $|T \cap\{1,2,3\}| \leq 1$.

Knudsen shows that $D^{T}$ is a smooth divisor, and in fact is isomorphic to the product $\mathbf{X}_{|T|+1} \times \mathbf{X}_{\left|T^{C}\right|+1}$ (the branch point counts as an "extra" point for each factor).

$$
\pi: \mathbf{X}_{n+1} \rightarrow \mathbf{X}_{n}
$$

factors through

$$
\pi_{1}: \mathbf{X}_{n+1} \rightarrow \mathbf{X}_{n} \times \mathbf{X}_{4}
$$


where $\pi_{1}$ is induced by $\pi$ and the map

$$
\pi_{1,2,3, n+1}: \mathbf{X}_{n+1} \rightarrow \mathbf{X}_{4}
$$

which is obtained by composing contractions, in such a way that every section but the first, second, third, and $(n+1)$ th is contracted. Let $B_{1}=\mathbf{X}_{n} \times \mathbf{X}_{4}$. The universal sections $\sigma^{1}, \sigma^{2}, \ldots, \sigma^{n}$ of $\pi$ induce sections

$$
\sigma_{1}^{1}=\pi_{1} \circ \sigma^{1}, \ldots, \sigma_{1}^{n}=\pi_{1} \circ \sigma^{n}
$$

of $B_{1}$. Embed $D^{T} \hookrightarrow B_{1}$ as $\sigma_{1}^{i}\left(D^{T}\right)$ for any $i \in T$ (we show that the restrictions of $\sigma_{1}^{i}$ and $\sigma_{1}^{j}$ to $D^{T}$ are the same for any $i, j \in T$ ) and let $B_{2}$ be the blowup of $B_{1}$ along the union of $D^{T}$ with $\left|T^{C}\right|=2$. (These turn out to be disjoint.) Inductively, having defined

$$
B_{k} \rightarrow B_{k-1} \rightarrow \cdots \rightarrow B_{1}
$$

we let $B_{k+1}$ be the blowup of $B_{k}$ along the union of the strict transforms of the $D^{T} \hookrightarrow B_{1}$, under $B_{k} \rightarrow B_{1}$, for which $\left|T^{C}\right|=k+1$. We prove inductively that these strict transforms are disjoint, and isomorphic to $D^{T}$. (Thus in each case we blow up along a disjoint union of codimension two subvarieties each of which is isomorphic to a product $\mathbf{X}_{i} \times \mathbf{X}_{j}$ for various $i, j<n$.) The key result of the chapter is that $\mathbf{X}_{n+1} \stackrel{p}{\longrightarrow} B_{1}$ is isomorphic to $B_{n-2} \rightarrow B_{1}$.

From this blowup description we prove the following:

(1) $\mathrm{cl}: \mathbf{A}_{*}\left(\mathbf{X}_{n}\right) \rightarrow H_{*}\left(\mathbf{X}_{n}\right)$ is an isomorphism, in particular, $\mathbf{X}_{n}$ has no odd homology and its Chow groups are finitely generated and free abelian.

(2) For any scheme $S, \mathbf{A}^{*}\left(\mathbf{X}_{n} \times S\right)$ is canonically isomorphic to $\mathbf{A}^{*}\left(\mathbf{X}_{n}\right) \otimes$ $\mathbf{A}^{*}(S)$.

$$
\mathbf{A}^{k}\left(\mathbf{X}_{n+1}\right) \stackrel{\Psi}{\cong} \mathbf{A}^{k}\left(\mathbf{X}_{n}\right) \oplus \mathbf{A}^{k-1}\left(\mathbf{X}_{n}\right) \otimes \bigoplus_{\substack{T \subset\{1,2, \ldots, n\} \\|T|,\left|T^{C}\right| \geq 2 \\|T \cap\{1,2,3\}| \leq 1}} \mathbf{A}^{k-1}\left(D^{T}\right)
$$

where $\Psi$ is induced by

$$
\begin{gathered}
\mathbf{A}^{k}\left(\mathbf{X}_{n}\right) \stackrel{\pi^{*}}{\longrightarrow} \mathbf{A}^{k}\left(\mathbf{X}_{n+1}\right), \\
\mathbf{A}^{k-1}\left(\mathbf{X}_{n}\right) \stackrel{\pi^{*}}{\longrightarrow} \mathbf{A}^{k-1}\left(\mathbf{X}_{n+1}\right) \stackrel{\Pi_{1,2,3, n+1}}{\longrightarrow} \mathbf{A}^{k}\left(\mathbf{X}_{n+1}\right), \\
\mathbf{A}^{k-1}\left(D^{T}\right) \stackrel{g^{*}}{\longrightarrow} \mathbf{A}^{k-1}\left(D^{T, n+1}\right) \stackrel{j_{*}}{\longrightarrow} \mathbf{A}^{k}\left(\mathbf{X}_{n+1}\right) .
\end{gathered}
$$

Here $\Pi_{1,2,3, n+1}$ indicates the first Chern class of the pullback of the canonical bundle under $\pi_{1,2,3, n+1}: \mathbf{X}_{n} \rightarrow \mathbf{X}_{4}$ that is

$$
\Pi_{1,2,3, n+1} \stackrel{\text { def }}{=} \pi_{1,2,3, n+1}^{*}\left(c_{1}(\mathscr{O}(1))\right)
$$

and $j$ and $g$ are defined by the commutative diagram:

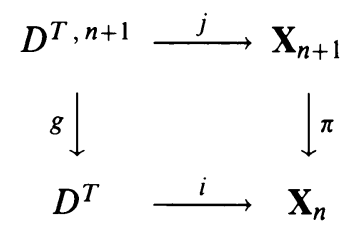


The inverse of $\Psi$ is $\left(\pi_{*} \circ \Pi_{1,2,3, n+1},-\pi_{*},-g_{*} j^{*}\right)$.

(4) The Chow groups $\mathbf{A}^{k}\left(\mathbf{X}_{n}\right)$ are free Abelian and their ranks

$$
a^{k} \stackrel{\text { def }}{=} \text { rank of } \mathbf{A}^{k}\left(\mathbf{X}_{n}\right)
$$

are given recursively by the formula

$$
\begin{gathered}
a^{k}(n+1)=a^{k}(n)+a^{k-1}(n)+\frac{1}{2} \sum_{j=2}^{n-2}\left(\begin{array}{l}
n \\
k
\end{array}\right) \sum_{l=0}^{l=k-1} a^{l}(j+1) a^{k-1-l}(n-j-1) . \\
a^{k}(3)= \begin{cases}1 & \text { if } k=0, \\
0 & \text { otherwise },\end{cases}
\end{gathered}
$$

for the particular case of divisors we have

$$
a^{1}(n)=2^{n-1}-\left(\begin{array}{l}
n \\
2
\end{array}\right)-1 .
$$

(5) Via $\Psi$ dual bases for $\mathbf{A}^{*}\left(\mathbf{X}_{n}\right)$ and $\mathbf{A}^{*}\left(D^{T}\right)$ induce dual bases for $\mathbf{A}^{*}\left(\mathbf{X}_{n+1}\right)$ as follows:

Let $m=n-2$ be the dim of $\mathbf{X}_{n+1}$ and let

$$
\begin{gathered}
\alpha_{1} \in \mathbf{A}^{k}\left(\mathbf{X}_{n}\right), \quad \alpha_{2} \in \mathbf{A}^{k-1}\left(\mathbf{X}_{n}\right), \quad \alpha_{3} \in \mathbf{A}^{k-1}\left(D^{T}\right), \\
\beta_{1} \in \mathbf{A}^{m-k}\left(\mathbf{X}_{n}\right), \quad \beta_{2} \in \mathbf{A}^{m-k-1}\left(\mathbf{X}_{n}\right), \quad \beta_{3} \in \mathbf{A}^{m-k-1}\left(D^{T}\right) ;
\end{gathered}
$$

then we have a multiplication table in $\mathbf{A}^{*}\left(\mathbf{X}_{n+1}\right)$,

$$
\left\|\begin{array}{cccc}
\cdot & \Psi\left(\alpha_{2}\right) & \Psi\left(\alpha_{1}\right) & \Psi\left(\alpha_{3}\right) \\
\Psi\left(\beta_{1}\right) & \alpha_{2} \cdot \beta_{1} & 0 & 0 \\
\Psi\left(\beta_{2}\right) & 0 & \alpha_{1} \cdot \beta_{2} & 0 \\
\Psi\left(\beta_{3}\right) & 0 & 0 & \alpha_{3} \cdot \beta_{3}
\end{array}\right\| .
$$

Furthermore if $\gamma \in \mathbf{A}^{k-1}\left(D^{T}\right)$ and $\delta \in \mathbf{A}^{m-k-1}\left(D^{S}\right)$ with $T \neq S$ then $\Psi(\gamma)$. $\Psi(\delta)=0$.

(6)

$$
\mathbf{A}^{*}\left(\mathbf{X}_{n}\right)=\frac{\mathbf{Z}\left[D^{S}|S \subset\{1,2, \ldots, n\}| S|,| S^{C} \mid \geq 2\right]}{\text { the following relations }} .
$$

(1) $D^{S}=D^{S^{C}}$,

(2) For any four distinct elements $i, j, k, l \in\{1,2, \ldots, n\}$ :

$$
\sum_{\substack{i, j \in S \\ k, l \notin S}} D^{S}=\sum_{\substack{i, k \in S \\ j, l \notin S}} D^{S}=\sum_{\substack{i, l \in S \\ j, k \notin S}} D^{S} .
$$

(3) $D^{S} D^{T}=0$ unless one of the following holds:

$$
S \subset T, \quad T \subset S, \quad S \subset T^{C}, \quad T^{C} \subset S .
$$

Under the isomorphism, $D^{S}$ is sent to the class of the corresponding vital divisor, while the three sums of (2) are the pullbacks of the vital divisors

$$
D^{i, j}, D^{i, k}, D^{i, l} \hookrightarrow \mathbf{X}_{4}
$$

under the morphism $\pi_{i, j, k, l}: \mathbf{X}_{n} \rightarrow \mathbf{X}_{4}$ which contracts all the sections except for the $i$ th, $j$ th, $k$ th and $l$ th. 
With this understanding these relations have the following "geometric" content:

(1) This corresponds to the fact that $D^{S}$ and $D^{S^{C}}$ are the same divisor.

(2) $\mathbf{X}_{4}$ is isomorphic to $\mathbf{P}^{1}$ and the three vital divisors $D^{i, j}, D^{i, k}$ and $D^{i, l}$ are points. The relation thus corresponds to the fact that the three points are linearly equivalent in $\mathbf{P}^{1}$.

(3) This relation expresses the fact that the divisors $D^{S}$ and $D^{T}$ are disjoint unless one of the stated conditions holds.

I wish to thank Bill Fulton for bringing this space to my attention and for providing considerable advice and encouragement throughout my investigations.

The paper is organized as follows. We begin with a catalogue of useful results on the vital divisors. Section 1, which is the heart of the chapter, contains our alternate construction of $\mathbf{X}_{n}$. In $\S 2$ we study the cl map, obtaining results (1) and (2). In $\S 3$ we obtain (3), (4) and (5). In $\S 4$ we obtain (6), our expression for $\mathbf{A}^{*}\left(\mathbf{X}_{n}\right)$. In the appendix a number of general results on the Chow rings of regular blowups are developed. Within a section the results are numbered beginning with one while for example in $\S 3$, the second theorem of $\S 1$ would be referred to as Theorem 1.2.

\section{Catalogue of Results on the vital divisors $D^{T}$}

This section consists of a series of results dealing with the vital divisors which will be useful throughout the chapter. We will often consider the map $\mathbf{X}_{n} \rightarrow \mathbf{X}_{n-k}$ obtained by contracting some collection of $k$ sections. In case we contract $\sigma^{k+1}, \sigma^{k+2}, \ldots, \sigma^{n}$ we will sometimes denote the map

$$
\{1,2,3 \ldots, n\} \rightarrow\{1,2,3, \ldots, k\} .
$$

Of particular interest are the maps

$$
\mathbf{X}_{n} \stackrel{\pi_{i, j, k, l}}{\longrightarrow} \mathbf{X}_{4}
$$

(for $i, j, k, l$ four distinct elements of $1,2, \ldots, n$ ), which contract every section but the $i$ th, $j$ th, $k$ th, and $l$ th. We will most often consider the maps $\pi_{1,2,3, i}$ which we denote $\phi^{i}$, or $\phi_{m}^{i}$ if we wish to make clear that the domain is $\mathbf{X}_{m}$. We also define $\phi^{1}, \phi^{2}$ and $\phi^{3}$ to be the constant maps

$$
\phi^{j}=D^{j, 4} \hookrightarrow \mathbf{X}_{4} \cong \mathbf{P}^{1}
$$

for $j$ between one and three. Recall from the introduction that $D_{n}^{T} \stackrel{i_{T}}{\hookrightarrow} \mathbf{X}_{n}$ is the divisor whose generic element consists of curves with two branches, the points of $T$ on one branch and the points of $T^{C}$ on the other. We always assume that $|T|,\left|T^{C}\right| \geq 2$.

Fact 1. The collection of $D^{T}$ is a family of smooth divisors in $\mathbf{X}_{n}$ with normal crossings.

Proof. See [Kn, Theorem 2.7].

Fact 2.

$$
D_{n}^{T} \cong \mathbf{X}_{|T|+1} \times \mathbf{X}_{\left|T^{c}\right|+1}
$$

via the restriction to $D_{n}^{T}$ of the map

$$
\mathbf{X}_{n} \rightarrow \mathbf{X}_{|T|+1} \times \mathbf{X}_{\left|T^{c}\right|+1}
$$


which is the product of contracting all but one section of $T^{C}$ and of contraction all but one section of $T$. (The restriction is independent of which two sections we choose not to contract, in any case the effect is to choose the branch point.)

Furthermore, if we label the points of the $\mathbf{X}_{|T|+1}$ factor by the points of $T \cup\{b\}$ with the "extra" section going to $b$ and the points of the $\mathbf{X}_{\left|T^{c}\right|+1}$ factor by the points of $T^{C} \cup\{b\}$ then the restriction of $\pi_{i, j, k, l}$ to $D_{n}^{T}$ is

$$
\begin{array}{cl}
\pi_{i, j, k, l} \circ p_{1} & \text { if } i, j, k, l \in T, \\
\pi_{i, j, k, l} \circ p_{2} & \text { if } i, j, k, l \in T^{C}, \\
\pi_{i, j, k, b} \circ p_{1} & \text { if } i, j, k \in T \text { and } l \in T^{C}, \\
\pi_{i, j, k, b} \circ p_{2} & \text { if } i, j, k \in T^{C} \text { and } l \in T, \\
\text { constant } & \text { if }|\{i, j, k, l\} \cap T|=2 .
\end{array}
$$

(The other cases when $|\{i, j, k, l\} \cap T|=3$ or $\left|\{i, j, k, l\} \cap T^{C}\right|=3$ are described analogously.)

Proof. See [Kn, Theorem 3.7].

Remark. In particular Fact 2 shows that the inclusion of $D^{T}$ in $\mathbf{X}_{n}$ has a section and thus pullback and pushforward and Chow groups are surjective and injective respectively.

For two subsets $S$ and $T$ of $\{1,2,3, \ldots, n\}$ we write $S * * T$ iff one of the following holds:

$$
S \subset T, \quad T \subset S, \quad T \cap S=\varnothing, \quad T \cup S=\{1,2,3, \ldots, n\} .
$$

(Observe that if we assume $|T \cap\{1,2,3\}| \leq 1$ and $|S \cap\{1,2,3\}| \leq 1$ the last equality is impossible.)

Fact 3. For some subset $I$ of $\{1,2,3, \ldots, n\}$ with $n-k$ elements let

$$
\mathbf{X}_{n} \stackrel{\pi_{I}}{\longrightarrow} \mathbf{X}_{n-k}
$$

contract the elements of $I^{C}$. Then

$$
\pi_{I}^{*}\left(D_{n-k}^{T}\right)=\sum_{\substack{T \subset S \\ S \subset T \cup I^{C}}} D_{n}^{S} .
$$

Proof. By induction it is enough to consider

$$
\mathbf{X}_{n+1} \stackrel{\pi}{\longrightarrow} \mathbf{X}_{n}
$$

contracting the $(n+1)$ th section. It is clear that $\pi^{*}\left(D_{n}^{T}\right)$ is a sum of vital divisors and it is clear from the pointwise description of the contraction map given in the introduction that only $D_{n+1}^{T}$ and $D_{n+1}^{T, n+1}$ appear in the sum. Finally, Knudsen's Theorem 2.7 shows that $\pi$ has geometrically reduced fibers (a local description of $\pi$ is given in the introduction) and hence $\pi^{*}\left(D_{n}^{T}\right)$ is reduced. It follows that

$$
\pi^{*}\left(D_{n}^{T}\right)=D_{n+1}^{T, n+1}+D_{n+1}^{T}
$$

which completes the proof.

Fact 4.

$$
D^{T} \cap D^{S} \neq \varnothing \quad \text { iff } \quad T * * S
$$


and in this case

$$
\begin{aligned}
D^{T} \cap D^{S} & =D_{|T|+1}^{S} \times \mathbf{X}_{\left|T^{C}\right|+1} & & \text { if } S \subset T \\
& =D_{|T|+1}^{S^{C}} \times \mathbf{X}_{\left|T^{C}\right|+1} & & \text { if } S^{C} \subset T \\
& =\mathbf{X}_{|T|+1} \times D_{\left|T^{C}\right|+1}^{S} & & \text { if } S \subset T^{C} \\
& =\mathbf{X}_{|T|+1} \times D_{\left|T^{C}\right|+1}^{S^{C}} & & \text { if } S^{C} \subset T^{C} .
\end{aligned}
$$

Proof. For the first point we show that if $T$ does not ** $S$ then $D^{T} \cap D^{S}$ is empty. By assumption there are elements $i, j, k, l \in\{1,2,3, \ldots, n\}$, $i \in T \backslash S, j \in S \backslash T, k \in T \cap S, l \notin T \cup S$. Then by Fact $3 \pi_{i, j, k, l}$ sends $D^{T}$ to $D_{4}^{i, k}$ and sends $D^{S}$ to $D_{4}^{j, k}$. As $D_{4}^{i, k}$ and $D_{4}^{j, k}$ are distinct points of $\mathbf{P}^{1}$ it follows that $D^{T}$ and $D^{S}$ are disjoint.

For the second point we may assume $T=\{1,2, \ldots, j\}$ and that $S \varsubsetneqq T$. Consider the commutative diagram

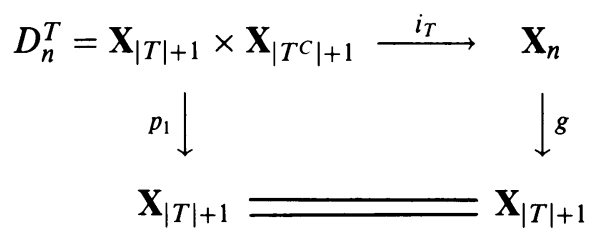

where $g$ is the map

$$
\{1,2,3, \ldots, n\} \rightarrow\{1,2,3, \ldots, j+1\} .
$$

By Fact 3

$$
g^{*}\left(D_{j+1}^{S}\right)=D_{n}^{S}+\sum_{\substack{S \subset V \\ V \subset S \cup\{j+2, j+3, \ldots, n\}}} D_{n}^{V} .
$$

But by the first part of Fact 4 each element in the sum is disjoint from $D^{T}$. (For any such $V$ we have $S \subset V \cap T, j+1 \notin T \cup V, V \cap\{j+1, j+2, \ldots, n\} \neq \varnothing$ so $V \nsubseteq T, V \cap T=S \neq T$ so $T \nsubseteq V$ ); thus

$$
i_{T}^{*}\left(D_{n}^{S}\right)=p_{1}^{*}\left(D_{j+1}^{S}\right)=D_{|T|+1}^{S} \times \mathbf{X}_{\left|T^{C}\right|+1}
$$

as required.

Fact 5. For any subset $I \subset\{1,2,3, \ldots, n\}$ the scheme $\phi^{i}=\phi^{j}$ for all $i, j \in I$ is the sum of the divisors

$$
\sum_{\substack{I \subset T \\|T \cap\{1,2,3\}| \leq 1}} D_{n}^{T} .
$$

Proof. One easily reduces (using the above facts) to the case where $I$ has only two elements. If both are in $\{1,2,3\}$ then the sum is empty, as expected. If one of these is 1,2 , or 3 , then we may assume $I=\{1,4\}$. The scheme $\phi^{1}=\phi^{4}$ is

$$
\pi_{1,2,3,4}^{-1}\left(D_{4}^{1,4}\right)=\sum_{\substack{1,4 \in T \\ 2,3 \notin T}} D_{n}^{T}
$$


by Fact 3, which is the desired result. For the final case we may thus assume $I=\{4,5\}$. Consider the commutative diagram

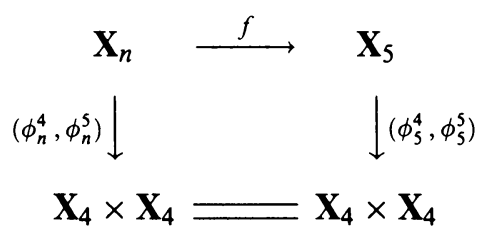

where $f$ is the map

$$
\{1,2,3, \ldots, n\} \rightarrow\{1,2,3,4,5\} .
$$

The scheme $\phi_{n}^{4}=\phi_{n}^{5}$ is $\left(\phi_{n}^{4}, \phi_{n}^{5}\right)^{*}(\delta)$ where $\delta$ is the diagonal. One can check explicitly (by using Knudsen's method of constructing $\mathbf{X}_{5}$ ) that

$$
\left(\phi_{5}^{4}, \phi_{5}^{5}\right)^{*}(\delta)=D_{5}^{4,5}+D_{5}^{1,4,5}+D_{5}^{2,4,5}+D_{5}^{3,4,5}
$$

and the result follows by applying $f^{*}$ according to Fact 3 .

\section{DeSCRIPTION OF $\mathbf{X}_{n}$ AS A COMPOSITION OF SMOOTH blowUPS}

In this section, whenever we indicate a subset

$$
T \subset\{1,2,3, \ldots, n\} .
$$

We will assume that $|T \cap\{1,2,3\}| \leq 1$.

$$
\mathbf{X}_{n+1} \stackrel{\pi}{\longrightarrow} \mathbf{X}_{n}
$$

factors as

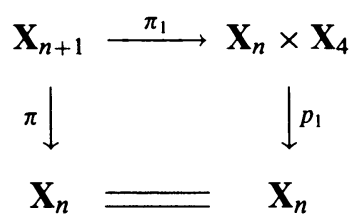

where $p_{1}$ is the projection onto the first factor and $\pi_{1}$ is induced by $\pi$ and $\phi_{n+1}^{n+1}$. Observe that

$$
\phi_{n+1}^{i}=\phi_{n}^{i} \circ \pi \quad \text { for } i \in\{1,2,3, \ldots, n\}
$$

and $\phi_{n+1}^{n+1}=p_{2} \circ \pi_{1}$ ( $p_{2}$ the projection onto the second factor). In view of this we will drop the subscripts, and write $\phi^{j}$. The domain scheme, which may be any of $\mathbf{X}_{n}, \mathbf{X}_{n+1}, \mathbf{X}_{n} \times \mathbf{X}_{4}$, or some intermediate scheme through which $\pi_{1}$ factors, will be clear from context. $\pi$ has $n$ universal sections $\sigma^{1}, \ldots, \sigma^{n}$ which induce sections $\sigma_{1}^{i}$ of $p_{1}$, with $p_{2} \circ \sigma_{1}^{i}=\phi^{i}$.

Lemma 1. The collection of

$$
D_{n+1}^{T, n+1} \hookrightarrow \mathbf{X}_{n+1}
$$

with

$$
T \subset\{1,2,3, \ldots, n\}
$$

are exactly the exceptional divisors of $\pi_{1}$. 
Proof. $\pi_{1}$ is an isomorphism away from the union of the vital divisors of $\mathbf{X}_{n+1}$ and these are of the form $D_{n+1}^{T}$ or $D_{n+1}^{T, n+1}$ or $D_{n+1}^{i, n+1}$. By Fact $2 \pi$ carries $D_{n+1}^{D, n+1}$ isomorphically onto $\mathbf{X}_{n}$ (in fact $D_{n+1}^{i, n+1}=\sigma^{i}\left(\mathbf{X}_{n}\right)$ ). By Fact 3

$$
\pi^{-1}\left(D_{n}^{T}\right)=D_{n+1}^{T}+D_{n+1}^{T, n+1}
$$

while by Fact $5 \phi^{n+1}$ agrees with $\phi^{i}$ on $D_{n+1}^{T, n+1}$ for any $i \in T$. This implies that

Also since

$$
\pi_{1}\left(D_{n+1}^{T, n+1}\right)=\sigma_{1}^{i}\left(D_{n}^{T}\right) .
$$

we necessarily have

$$
\pi_{1}^{-1}\left(D_{n}^{T} \times \mathbf{X}_{4}\right)=D_{n+1}^{T}+D_{n+1}^{T, n+1}
$$

$$
\pi_{1}\left(D_{n+1}^{T}\right)=D_{n}^{T} \times \mathbf{X}_{4}
$$

This completes the proof.

Inductively we now define schemes $B_{k}$ with subschemes

$$
\begin{aligned}
S_{k}^{T} & \text { for }\left|T^{C}\right| \geq k+1, \\
R_{k}^{T} & \text { for }\left|T^{C}\right| \geq k+1, \\
E_{k}^{T} & \text { for }\left|T^{C}\right| \leq k, \\
\Sigma_{k}^{i} & \text { for } i=1,2, \ldots, n,
\end{aligned}
$$

and maps

$$
B_{k+1} \stackrel{f_{k+1}}{\longrightarrow} B_{k} .
$$

For $k=1$ let

$$
\begin{aligned}
& B_{1}=\mathbf{X}_{n} \times \mathbf{X}_{4}, \\
& S_{1}^{T}=\sigma_{1}^{i}\left(D_{n}^{T}\right) \text { for any } i \in T, \\
& \Sigma_{1}^{i}=\sigma_{1}^{i}\left(\mathbf{X}_{n}\right) \text { for any } i \in T, \\
& R_{1}^{T}=\text { the scheme } \phi^{i}=\phi^{j} \text { for all } i, j \in T \cup\{n+1\} .
\end{aligned}
$$

Observe that

$$
R_{1}^{T}=\sigma_{1}^{l} \text { (the scheme } \phi^{i}=\phi^{i} \forall i, j \in T \text { ) for any } l \in T .
$$

In particular $R_{1}^{T} \hookrightarrow \Sigma_{1}^{i}$ for any $i \in T$ and by Fact 2 as a subscheme of $\Sigma_{1}^{i}$ it is the sum of divisors

$$
R_{1}^{T}=\sum_{T \subset S} D_{n}^{S}
$$

Having defined this data for $k$ let

$$
B_{k+1} \stackrel{f_{k+1}}{\longrightarrow} B_{k}
$$

be the blowup of $B_{k}$ along the union of $S_{k}^{T}$ with $\left|T^{C}\right|=k+1$. (We will show that these $S_{k}^{T}$ are disjoint.) Let $S_{k+1}^{T}$ for $\left|T^{C}\right| \geq k+2$ and $\Sigma_{k+1}^{i}$ for $i=$ $1,2, \ldots, n$ be the strict transforms (under $f_{k+1}$ ) of $S_{k}^{T}$ and $\Sigma_{k}^{i}$ respectively. (Thus they are the strict transforms under the composition

$$
B_{k+1} \rightarrow B_{k} \rightarrow \cdots \rightarrow B_{1}
$$

of $S_{1}^{T}$ and $\Sigma_{1}^{i}$ respectively.) Let

$$
E_{k+1}^{T}=f_{k+1}^{-1}\left(S_{k}^{T}\right) \text { for }\left|T^{C}\right|=k+1
$$


and

$$
E_{k+1}^{T}=f_{k+1}^{-1}\left(E_{k}^{T}\right) \text { for }\left|T^{C}\right| \leq k .
$$

(Thus the $E_{k}^{T}$ are the exceptional divisors of $B_{k} \rightarrow B_{1}$.) Let $R_{k+1}^{T}$ be the residual scheme

$$
\mathscr{R}\left(\sum_{\substack{T \subset S \\\left|S^{C}\right| \leq k+1}} E_{k+1}^{S}, \phi^{i}=\phi^{j} \forall i, j \in T \cup\{n+1\}\right) .
$$

(In general, if we have a scheme $Y$ with a subscheme $X$ and a cartier divisor $D$ of $Y$ which is itself a subscheme of $X$

$$
D \hookrightarrow X \hookrightarrow Y
$$

then we obtain a scheme $\mathscr{R}=\mathscr{R}(D, X)$ the residual scheme to $D$ in $X$, we locally dividing equations for $X$ by a defining function for $D$. In terms of the ideal sheaves, $\mathscr{R}$ is characterized by the equation

$$
\mathscr{I}_{\mathscr{R}} \cdot \mathscr{I}_{D}=\mathscr{I}_{X} \text {.) }
$$

Observe that

$$
S_{1}^{T} \hookrightarrow \Sigma_{1}^{i} \quad \text { for any } i \in T
$$

It follows that

$$
S_{k}^{T} \hookrightarrow \Sigma_{k}^{i} \quad \text { for any } i \in T
$$

$\left(|T|^{C} \geq k+1\right)$.

With this notation the key result of the chapter is the following:

Theorem 1. The following hold for all $k$ :

(1) $\pi_{1}$ factors through $B_{k}$

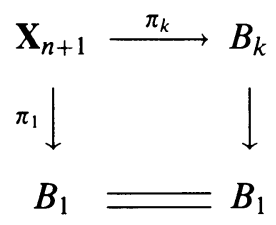

and so in particular $B_{k} \rightarrow B_{1}$ has sections $\sigma_{k}^{1}, \sigma_{k}^{2}, \ldots, \sigma_{k}^{n}$ induced by the universal sections $\sigma^{1}, \sigma^{2}, \ldots, \sigma^{n}$.

(2) $\Sigma_{k}^{i}=\sigma_{k}^{i}\left(\mathbf{X}_{n}\right) \cong \mathbf{X}_{n}$.

(3) $S_{k}^{T}=\sigma_{k}^{i}\left(D_{n}^{T}\right)$ for any $i \in T$.

(4) The $S_{k}^{T}$ with $\left|T^{C}\right|=k+1$ are disjoint. $k$.

(5) $\pi_{k}^{-1}\left(S_{k}^{T}\right)=D_{n+1}^{T, n+1}$ for $\left|T^{C}\right|=k+1$ and $\pi_{k}^{-1}\left(E_{k}^{s}\right)=D_{n+1}^{S, n+1}$ for $\left|S^{C}\right| \leq$

(6) $R_{k}^{T}$ is a subscheme of $\Sigma_{k}^{i}$ for all $i \in T$ and as a subscheme it is the sum of the divisors

$$
\sum_{\substack{T \subset S \\\left|S^{C}\right| \geq k+1}} D_{n}^{S} .
$$

The proof will require two lemmas. 
Lemma A. If $X \stackrel{f}{\longrightarrow} Y$ is a map of schemes and

$$
D \hookrightarrow W \hookrightarrow Y
$$

is a composition of subschemes of $Y$ with $D$ a cartier divisor of $Y$ then we have an equality of subschemes of $X$ :

$$
\mathscr{R}\left(f^{-1}(D), f^{-1}(W)\right)=f^{-1}(\mathscr{R}(D, W)) .
$$

Proof. This is immediate from the definitions: By assumption we have the equality of ideal sheaves on $Y$

$$
\mathscr{I}_{\mathscr{R}(D, W)} \cdot \mathscr{I}_{D}=\mathscr{I}_{W}
$$

which implies the equality of ideal sheaves on $X$

$$
f^{-1}\left(\mathscr{I}_{\mathscr{R}(D, W)}\right) \cdot f^{-1}\left(\mathscr{I}_{D}\right)=f^{-1}\left(\mathscr{I}_{W}\right)
$$

as required.

The second lemma requires a definition:

Definition. A subscheme $X \stackrel{i}{\hookrightarrow} Y$ with ideal sheaf $I \subset \mathscr{O}_{Y}$ is said to be linearly embedded if the canonical surjection from the $d$ th symmetric power of $I$ to $I^{d}$

$$
\operatorname{Sym}_{A}^{d}(I) \rightarrow I^{d}
$$

is an isomorphism for all $d$.

Linear embeddings are studied in [Ke2].

Lemma B. If $\left\{D^{i}\right\}_{i \in I}$ is a family of cartier divisors with normal crossings, then for any $j \in I$ the embedding

$$
D^{j} \hookrightarrow \sum_{i \in I} D^{i}
$$

is a linear embedding.

Proof. The question is local and follows from the following:

Sublemma. If $f_{1}, f_{2}, \ldots, f_{m}, g$ is a regular sequence in a ring $A$. Then the embedding

$$
V(g) \hookrightarrow V\left(f_{1} \cdot f_{2} \cdots \cdot f_{m} \cdot g\right)
$$

is linear.

Proof of the Sublemma. Since $g, f_{1} \cdot f_{2} \cdots \cdots f_{m}$ is a regular sequence we may assume that $m=1$. In any ring, a principal ideal $(g)$ is of linear type if and only if the annihilator of $g^{d}$ is the same as the annihilator of $g$. Thus to show that

$$
V(g) \hookrightarrow V(f \cdot g)
$$

is a linear embedding we need only check that modulo $f \cdot g$ the annihilators of $g$ and $g^{d}$ are the same. This follows easily from the definition of a regular sequence.

The proof of Theorem 1 relies on the following result:

Result. Let

$$
X \stackrel{i}{\hookrightarrow} Y \stackrel{j}{\hookrightarrow} Z
$$


be a composition of embeddings with $i$ linear. Let

$$
\tilde{Z} \stackrel{\pi}{\longrightarrow} Z
$$

be the blowup of $Z$ along $X$ and let $\tilde{Y}$ be the blowup of $Y$. Then $\tilde{Y}$ is the residual scheme to the exceptional divisor in $\pi^{-1}(Y)$,

$$
\tilde{Y}=\mathscr{R}\left(E, \pi^{-1}(Y)\right)
$$

Proof. This is a special case of [Ke2] Theorem 1.

Proof of Theorem 1. We proceed by induction. $k=1$ :

$(1)_{1},(2)_{1},(3)_{1}$ and $(6)_{1}$ have already been observed. When $\left|T^{C}\right|=2$, $S_{1}^{T}=R_{1}^{T}$ and

$$
\begin{aligned}
\pi_{1}^{-1}\left(S_{1}^{T}\right) & =\pi_{1}^{-1}\left(R_{1}^{T}\right) \\
& =\text { the scheme } \phi^{i}=\phi^{j} \forall i, j \in T \cup\{n+1\}, \\
& =D_{n+1}^{T, n+1} .
\end{aligned}
$$

(By Fact 5

$$
\text { the scheme } \begin{aligned}
\phi^{i} & =\phi^{j} \forall i, j \in T \cup\{n+1\}, \\
& =\sum_{\substack{S \subset\{1,2, \ldots, n+1\} \\
T, n+1 \subset S}} D_{n+1}^{S} .
\end{aligned}
$$

But since $\left|T^{C}\right|=2$ if $T, n+1 \subset S$ then $S=T \cup\{n+1\}$.) This establishes $(5)_{1}$. Since $D_{n+1}^{T, n+1}$ and $D_{n+1}^{S, n+1}$ are disjoint in $\mathbf{X}_{n+1}$ if $|T|=|S|$ (by Fact 4) it also establishes $(4)_{1}$.

Now for the induction step, we assume the theorem for $k$. Notice that $(5)_{k}$ implies $(1)_{k+1}$ since it shows that the inverse image under $\pi_{k}$ of the locus blown up by

$$
B_{k+1} \stackrel{f_{k+1}}{\longrightarrow} B_{k}
$$

is a divisor of $\mathbf{X}_{n+1} \cdot(1)_{k+1}$ implies $(2)_{k+1}$ and (3) $k+1$. Also $(5)_{k+1}$ implies $(4)_{k+1}$ as above. It remains to establish $(5)_{k+1}$ and $(6)_{k+1}$.

We begin with $(6)_{k+1}$. We have for $\left|T^{C}\right| \geq k+2$

$$
\begin{aligned}
R_{k+1}^{T} & =\mathscr{R}\left(\sum_{\substack{T \subset V \\
\left|V^{C}\right| \leq k+1}} E_{k+1}^{V}, \phi^{i}=\phi^{j} \forall i, j \in T \cup\{n+1\}\right) \\
& =\mathscr{R}\left(\sum_{\substack{T \subset V \\
V^{C} \mid=k+1}} E_{k+1}^{V}, \mathscr{R}\left(\sum_{\substack{T \subset V \\
V^{C} \mid \leq k}} E_{k+1}^{V}, \phi^{i}=\phi^{j} \forall i, j \in T \cup\{n+1\}\right),\right.
\end{aligned}
$$




$$
\begin{aligned}
& \mathscr{R}\left(\sum_{\substack{T \subset V \\
\left|V^{C}\right| \leq k}} E_{k+1}^{V}, \phi^{i}=\phi^{j} \forall i, j \in T \cup\{n+1\}\right) \\
& =\mathscr{R}\left(f_{k+1}^{-1}\left(\sum_{\substack{T \subset V \\
\left|V^{C}\right| \leq k}} E_{k}^{V}\right), f_{k+1}^{-1}\left(\phi^{i}=\phi^{j} \forall i, j \in T \cup\{n+1\}\right)\right)
\end{aligned}
$$

which by Lemma $\mathrm{A}$ is

$$
f_{k+1}^{-1}\left(\mathscr{R}\left(\sum_{\substack{T \subset V \\\left|V^{C}\right| \leq k}} E_{k+1}^{V}, \phi^{i}=\phi^{j} \forall i, j \in T \cup\{n+1\}\right)\right)=f_{k+1}^{-1}\left(R_{k}^{T}\right) .
$$

Also

$$
\sum_{\substack{T \subset V \\\left|V^{C}\right|=k+1}} E_{k+1}^{V}=f_{k+1}^{-1}\left(\bigcup_{\substack{T \subset V \\\left|V^{C}\right|=k+1}} S_{k}^{V}\right)
$$

Thus

$$
R_{k+1}^{T}=\mathscr{R}\left(f_{k+1}^{-1}\left(\bigcup_{\substack{T \subset V \\\left|V^{C}\right|=k+1}} S_{k}^{V}\right), f_{k+1}^{-1}\left(R_{k}^{T}\right)\right)
$$

Now, by $(6)_{k}$

is the sum of divisors

$$
R_{k}^{T} \hookrightarrow \Sigma_{k}^{i}=\sigma_{k}^{i}\left(\mathbf{X}_{n}\right) \quad \text { for any } i \in T
$$

$$
\sum_{\substack{T \subset S \\\left|S^{C}\right| \geq k+1}} D_{n}^{S}
$$

and

$$
\bigcup_{\substack{T \subset V \\ V^{C} \mid=k+1}} S_{k}^{V} \hookrightarrow \Sigma_{k}^{i}=\sigma_{k}^{i}\left(\mathbf{X}_{n}\right)
$$

is the sum of the disjoint divisors

$$
\sum_{\substack{T \subset V \\\left|V^{C}\right|=k+1}} D_{n}^{V}
$$

Thus, by Lemma B

$$
\bigcup_{\substack{T \subset V \\\left|V^{C}\right|=k+1}} S_{k}^{V} \hookrightarrow R_{k}^{T}
$$

is a linear embedding and so by the result stated above $R_{k+1}^{T}$ is the blowup of $R_{k}^{T}$ along

$$
\bigcup_{\substack{T \subset V \\\left|V^{C}\right|=k+1}} S_{k}^{V}
$$


or in terms of subschemes of $\Sigma_{k}^{i}$, the blowup of

$$
\sum_{\substack{T \subset S \\\left|S^{C}\right| \geq k+1}} D_{n}^{S}
$$

along

$$
\sum_{\substack{T \subset V \\\left|V^{C}\right|=k+1}} D_{n}^{V}
$$

which is clearly

$$
\sum_{\substack{T \subset S \\\left|S^{C}\right| \geq k+2}} D_{n}^{S} \hookrightarrow \Sigma_{k+1}^{i}=\sigma_{k}^{i}\left(\mathbf{X}_{n}\right)
$$

(for any $i \in T$ ).

This establishes $(6)_{k+1}$. For $(5)_{k+1}$ : If $\left|S^{C}\right| \leq k+1$ then

$$
\begin{aligned}
\pi_{k+1}^{-1}\left(E_{k+1}^{S}\right) & =\pi_{k+1}^{-1}\left(f_{k+1}^{-1}\left(E_{k}^{S}\right)\right)=\pi_{k}^{-1}\left(E_{k}^{S}\right) \\
& =D_{n+1}^{S, n+1} \quad \text { (by induction). }
\end{aligned}
$$

If $\left|T^{C}\right|=k+2$ then $(6)_{k+1}$ implies in particular that $S_{k+1}^{T}=R_{k+1}^{T}$ and so

$$
\pi_{k+1}^{-1}\left(S_{k+1}^{T}\right)=\pi_{k+1}^{-1}\left(R_{k+1}^{T}\right)
$$

which by Lemma $\mathrm{A}$ is equal to

$$
\mathscr{R}\left(\pi_{k+1}^{-1}\left(\sum_{\substack{T \subset S \\\left|S^{C}\right| \leq k+1}} E_{k+1}^{S}\right), \phi^{i}=\phi^{j} \forall i, j \in T \cup\{n+1\}\right) .
$$

By Fact 5 and $(*)$ this last expression is

$$
=\mathscr{R}\left(\sum_{\substack{T \subset S \\\left|S^{C}\right| \leq k+1}} D_{n+1}^{S, n+1}, \sum_{T \subset S} D_{n+1}^{S, n+1}\right)=D_{n+1}^{T, n+1} .
$$

This completes the proof.

An immediate corollary of Theorem 1 is

\section{Theorem 2.}

$$
\mathbf{X}_{n+1} \stackrel{\pi_{n-2}}{\longrightarrow} B_{n-2}
$$

is an isomorphism.

Proof. Observe first that by (3) $B_{k+1}$ is obtained from $B_{k}$ by blowing up along a smooth subvariety, and hence each of the $B_{k}$ are smooth. By Lemma 1, the only possible exceptional divisors of $\pi_{n-2}$ are the divisors $D_{n+1}^{T, n+1}$ but by (5),

$$
\pi_{n-2}\left(D_{n+1}^{T, n+1}\right)=E_{n-2}^{T}
$$

and $E_{n-2}^{T}$ has the same dimension as $D_{n+1}^{T, n+1}$. Thus $\pi_{n-2}$ has no exceptional divisors, and hence is an isomorphism. 
Notice that since $\mathbf{X}_{4}=\mathbf{P}^{1}$. Theorems 1 and 2 in particular exhibit $\mathbf{X}_{n}$ as a composition of smooth blowups of

$$
\mathbf{P}^{1} \times \mathbf{P}^{1} \times \cdots \times \mathbf{P}^{1}
$$

\section{Homological RESUlts}

Definition. A scheme of characteristic zero is said to be an HI (for homology isomorphism) scheme if the canonical map from the Chow groups to homology is an isomorphism.

Theorem 1. If $Y$ is an HI scheme then so is $Y \times \mathbf{X}_{n}$.

Proof. We proceed by induction. $\mathbf{X}_{4}$ is isomorphic to $\mathbf{P}^{1}$ and so the result is clear. Now assume the theorem for all $k$ less than or equal to $n$. By Theorem 1.2 the map

$$
Y \times \mathbf{X}_{n+1} \rightarrow Y \rightarrow \mathbf{X}_{n}
$$

is a composition of blowups along regularly embedded subvarieties each of which is isomorphic to

$$
Y \times \mathbf{X}_{i} \times \mathbf{X}_{j}
$$

for various $i, j$ less than or equal to $n-1$. In any case, by induction the base loci of each blowup is an HI scheme and the result follows from Theorem 2 of the appendix.

Theorem 2. For any scheme $Y$ the canonical map

$$
\mathbf{A}^{*}\left(\mathbf{X}_{n}\right) \otimes \mathbf{A}^{*}(Y) \rightarrow \mathbf{A}^{*}\left(\mathbf{X}_{n} \times Y\right)
$$

is an isomorphism.

Proof. We proceed by induction on $n$. The result is clear for $n$ equal to three or four. The general case follows by induction using Theorem 1.2 and the next lemma.

For the next lemma, we will say that a scheme $Y$ is simple if for any other scheme $S$ the canonical map

$$
\mathbf{A}^{*}(Y) \otimes \mathbf{A}^{*}(S) \rightarrow \mathbf{A}^{*}(Y \times S)
$$

is an isomorphism.

Lemma. If $X \stackrel{i}{\hookrightarrow} Y$ is regular embedding and $X$ and $Y$ are simple then so is $\tilde{Y}$ the blowup of $Y$ along $X$.

Proof. The result follows from the canonical exact sequence for the bivariant groups of a regular blowup [F, p. 333].

\section{BETTI NUMBERS AND DUAL BASIS}

Theorem 1. We have an isomorphism

$$
\mathbf{A}^{k}\left(\mathbf{X}_{n+1}\right) \stackrel{\Psi}{\cong} \mathbf{A}^{k}\left(\mathbf{X}_{n}\right) \oplus \mathbf{A}^{k-1}\left(\mathbf{X}_{n}\right) \oplus \bigoplus_{\substack{T \subset\{1,2, \ldots, n\} \\|T,| T^{C}|\geq 2\\| T \cap\{1,2,3\} \mid \leq 1}} \mathbf{A}^{k-1}\left(D^{T}\right)
$$


where $\Psi$ is induced by

$$
\begin{gathered}
\mathbf{A}^{k}\left(\mathbf{X}_{n}\right) \stackrel{\pi^{*}}{\longrightarrow} \mathbf{A}^{k}\left(\mathbf{X}_{n+1}\right), \\
\mathbf{A}^{k-1}\left(\mathbf{X}_{n}\right) \stackrel{\pi^{*}}{\longrightarrow} \mathbf{A}^{k-1}\left(\mathbf{X}_{n+1}\right) \stackrel{\Pi_{1,2,3, n+1}}{\longrightarrow} \mathbf{A}^{k}\left(\mathbf{X}_{n+1}\right), \\
\mathbf{A}^{k-1}\left(D^{T}\right) \stackrel{\boldsymbol{g}^{*}}{\longrightarrow} \mathbf{A}^{k-1}\left(D^{T, n+1}\right) \stackrel{j_{*}}{\longrightarrow} \mathbf{A}^{k}\left(\mathbf{X}_{n+1}\right) .
\end{gathered}
$$

Here $\Pi_{1,2,3, n+1}$ indicates the first Chern class of the pullback of the canonical bundle under $\pi_{1,2,3, n+1}: \mathbf{X}_{n} \rightarrow \mathbf{X}_{4}$ that is

$$
\Pi_{1,2,3, n+1} \stackrel{\text { def }}{=} \pi_{1,2,3, n+1}^{*}\left(c_{1}(\mathscr{O}(1))\right)
$$

and $j$ and $g$ are defined by the commutative diagram:

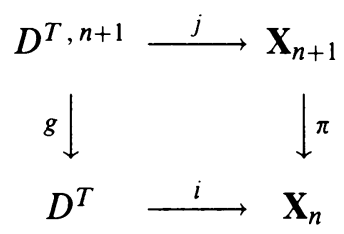

The inverse of $\Psi$ is $\left(\pi_{*} \circ \Pi_{1,2,3, n+1},-\pi_{*}, g_{*} j^{*}\right.$.)

Via $\Psi$ dual bases for $\mathbf{A}^{*}\left(\mathbf{X}_{n}\right)$ and $\mathbf{A}^{*}\left(D^{T}\right)$ induce dual bases for $\mathbf{A}^{*}\left(\mathbf{X}_{n+1}\right)$ as follows:

Let $m=n-2$ be the $\operatorname{dim}$ of $\mathbf{X}_{n+1}$ and let

$$
\begin{gathered}
\alpha_{1} \in \mathbf{A}^{k}\left(\mathbf{X}_{n}\right), \quad \alpha_{2} \in \mathbf{A}^{k-1}\left(\mathbf{X}_{n}\right), \quad \alpha_{3} \in \mathbf{A}^{k-1}\left(D^{T}\right), \\
\beta_{1} \in \mathbf{A}^{m-k}\left(\mathbf{X}_{n}\right), \quad \beta_{2} \in \mathbf{A}^{m-k-1}\left(\mathbf{X}_{n}\right), \quad \beta_{3} \in \mathbf{A}^{m-k-1}\left(D^{T}\right) ;
\end{gathered}
$$

then we have a multiplication table in $\mathbf{A}^{*}\left(\mathbf{X}_{n+1}\right)$

$$
\left\|\begin{array}{cccc}
\cdot & \Psi\left(\alpha_{2}\right) & \Psi\left(\alpha_{1}\right) & \Psi\left(\alpha_{3}\right) \\
\Psi\left(\beta_{1}\right) & \alpha_{2} \cdot \beta_{1} & 0 & 0 \\
\Psi\left(\beta_{2}\right) & 0 & \alpha_{1} \cdot \beta_{2} & 0 \\
\Psi\left(\beta_{3}\right) & 0 & 0 & \alpha_{3} \cdot \beta_{3}
\end{array}\right\| .
$$

Furthermore if $\gamma \in \mathbf{A}^{k-1}\left(D^{T}\right)$ and $\delta \in \mathbf{A}^{m-k-1}\left(D^{S}\right)$ with $T \neq S$ then $\Psi(\gamma) \cdot$ $\Psi(\delta)=0$.

Proof. The result follows essentially immediately from Theorem 1.2 and Theorem 3 of the appendix. From these theorems it follows that for each $k$ we have an isomorphism

$$
\mathbf{A}^{k}\left(B_{k}\right) \bigoplus_{\left|T^{C}\right|=k+1} \mathbf{A}^{k-1}\left(D_{n}^{T}\right) \stackrel{\Psi_{k}}{\longrightarrow} \mathbf{A}^{k}\left(B_{k+1}\right)
$$

via which dual bases for $B_{k}$ and $D_{n}^{T}$ induce dual bases for $B_{k+1}$. Induction then gives an isomorphism

$$
\mathbf{A}^{k}\left(B_{1}\right) \bigoplus_{T \subset\{1,2,3, \ldots, n\}} \mathbf{A}^{k-1}\left(D_{n}^{T}\right) \rightarrow \mathbf{A}^{k}\left(\mathbf{X}_{n+1}\right)
$$

via which dual bases are analogously induced. The isomorphism given in the theorem now follows from the fact that $B_{1}$ is $\mathbf{X}_{n} \times \mathbf{P}^{1}$. 


\section{Calculation of $\mathbf{A}^{*}\left(\mathbf{X}_{n}\right)$}

Until we reach the statement of Theorem 1 of this section we will always assume when considering $T \subset\{1,2,3, \ldots, n\}$ (in addition to the assumption which we are making throughout the chapter that $T$ and its complement have at least two elements) that $|T \cap\{1,2,3\}| \leq 1$. Also we will frequently be simultaneously discussing the vital divisors of $\mathbf{X}_{n}$ and $\mathbf{X}_{n+1}$. When we write $D_{n+1}^{T, n+1}$ we implicitly assume that $T \subset\{1,2,3, \ldots, n\}$.

Our calculations depend on our presentation of

$$
\mathbf{X}_{n+1} \cong B_{n-2}
$$

and the following lemma.

Lemma 1. Suppose that a subscheme $X \stackrel{i}{\hookrightarrow} Y$ of a scheme $Y$ is the complete intersection of two divisors $D_{1}$ and $D_{2}$ and that the pullback

$$
\mathbf{A}^{*}(Y) \stackrel{i^{*}}{\longrightarrow} \mathbf{A}^{*}(X)
$$

is surjective. Let $\tilde{Y} \stackrel{\pi}{\longrightarrow} Y$ be the blowup of $Y$ along $X$. Then the bivariant (also called Chow cohomology) ring of $\tilde{Y}$ is

$$
\mathbf{A}^{*}(\tilde{Y})=\frac{\mathbf{A}^{*}(Y)[T]}{\left(D_{1}-T\right)\left(D_{2}-T\right), T \cdot \operatorname{ker} i^{*}} .
$$

The isomorphism is induced by $\pi^{*}$ and by sending $T$ to the class of the exceptional divisor.

Proof. This is a special case of Theorem 1 of the appendix.

We will also need the following three lemmas:

Let $P_{k}^{T}$ be the strict transform of $S_{1}^{T} \times \mathbf{X}_{4}$ under

$$
B_{k} \rightarrow B_{1} \text {. }
$$

Lemma 2. For each $k$ and for any $i$ in $T, S_{k}^{T}$ is the complete intersection of the divisors $\Sigma_{k}^{i}$ and $P_{k}^{T}$. Furthermore the restriction

$$
\mathbf{A}^{*}\left(B_{k}\right) \rightarrow \mathbf{A}^{*}\left(S_{k}^{T}\right)
$$

is surjective.

Proof. By Theorem $1.1 \Sigma_{k}^{i}$ is isomorphic to $\mathbf{X}_{n}$ and this isomorphism realizes the subvariety $S_{k}^{T}$ of $\Sigma_{k}^{i}$ as the subvariety $D_{n}^{T}$ of $\mathbf{X}_{n}$. As remarked in the preliminaries after Fact 2 the restriction

$$
\mathbf{A}^{*}\left(\mathbf{X}_{n}\right) \rightarrow \mathbf{A}^{*}\left(D_{n}^{T}\right)
$$

is surjective. The restriction

$$
\mathbf{A}^{*}\left(B_{k}\right) \rightarrow \mathbf{A}^{*}\left(\Sigma_{k}^{i}\right)
$$

is also surjective (since the inclusion of $\Sigma_{k}^{i}$ in $B_{k}$ has a section given by the projection to $\mathbf{X}_{n}$ ). Thus the given restriction is a composition of surjections. 
The first remark is proved by induction. The case of $k=1$ is clear. We have (by induction) a commutative diagram

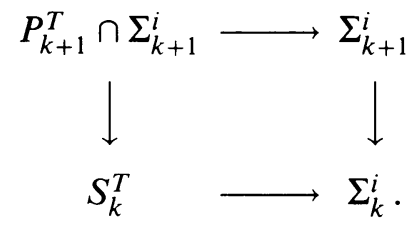

By Theorem 1.1 the map $\Sigma_{k+1}^{i} \rightarrow \Sigma_{k}^{i}$ is an isomorphism, carrying $S_{k+1}^{T}$ isomorphically onto $S_{k}^{T}$. The left column is an isomorphism generically on $S_{k}^{T}$. Thus $P_{k+1}^{T} \cap \Sigma_{k+1}^{i}$ is a subscheme of and generically equal to $S_{k+1}^{T}$. Since $S_{k+1}^{T}$ is integral it follows that the two are equal.

Lemma 3.

$$
\begin{aligned}
& f_{k+1}^{-1}\left(P_{k}^{T}\right)=P_{k+1}^{T} \quad \text { for }\left|T^{C}\right| \neq k+1, \\
& f_{k+1}^{-1}\left(P_{k}^{T}\right)=E_{k+1}^{T}+P_{k+1}^{T} \quad \text { for }\left|T^{C}\right|=k+1 .
\end{aligned}
$$

Proof. In any case

$$
f_{k+1}^{-1}\left(P_{k}^{T}\right)=P_{k+1}^{T}+\text { sum of various } E_{k+1}^{V} \text { with }\left|V^{C}\right|=k+1 .
$$

Further a particular $E_{k+1}^{V}$ appears in this sum if and only if $S_{k}^{V}$ is contained in $P_{k}^{T}$. By the previous lemma this holds if $V$ is equal to $T$ and since the images of the two subvarieties in $\mathbf{X}_{n}$ are $D_{n}^{V}$ and $D_{n}^{T}$ this is the only way it can hold.

Lemma 4. If $V^{C}$ consists of $k+1$ elements and $T^{C}$ of strictly more elements then $S_{k}^{V}$ meets $S_{k}^{T}$ if and only if $T$ is contained in $V$. In this case the intersection is a smooth cartier divisor of each.

Proof. If $T \subset V$ then by Theorem 1.1 (3), $S_{k}^{V}$ and $S_{k}^{T}$ are subvarieties of $\Sigma_{k}^{i}$ for any $i$ in $T$ and under the isomorphism of $\Sigma_{k}^{i}$ with $\mathbf{X}_{n}$ they correspond to the divisors $D_{n}^{V}$ and $D_{n}^{T}$ which intersect in a smooth cartier divisor of each by Fact 4 .

Now assume that $S_{k}^{V}$ and $S_{k}^{T}$ have nonempty intersection. Let $i$ be any element of $T$. We will show that it is in $V$. Necessarily $\Sigma_{k}^{i}$ meets $S_{k}^{V}$ and since $\Sigma_{k+1}^{i}$. is isomorphic to $\Sigma_{k}^{i}$ by Theorem 1.1(3), necessarily the intersection is a cartier divisor of $\Sigma_{k}^{i}$. We conclude by dimension considerations that $S_{k}^{V}$ is a subvariety of $\Sigma_{k}^{i}$ (otherwise the intersection is of pure codimension one in $S_{k}^{V}$ ), necessarily the subvariety $D_{n}^{V}$. Let $j$ be some element of $V$. In particular we conclude that

$$
\sigma_{1}^{i}\left(D_{n}^{V}\right)=\sigma_{1}^{j}\left(D_{n}^{V}\right)
$$

and thus $\phi^{i}$ and $\phi^{j}$ agree on $D_{n}^{V}$. (The above sections are the graphs of the restrictions of these maps to $D_{n}^{V}$.) It follows from Fact 5 that $i$ is in $V$ as required.

Claim.

$\mathbf{A}^{*}\left(B_{k+1}\right)=\frac{\mathbf{A}^{*}\left(B_{k}\right)\left[E_{k+1}^{T} \text { for }\left|T^{C}\right| \leq k+1, P_{k+1}^{T}, \Sigma_{k+1}^{i} \text { for } T, i \subset\{1, \ldots, n\}\right]}{\text { the following relations }}$.

(1) $P_{k+1}^{T}=P_{k}^{T}-E_{k+1}^{T}$ for $\left|T^{C}\right|=k+1$, 
(2) $P_{k+1}^{T}=P_{k}^{T}$ for $\left|T^{C}\right| \neq k+1$,

(3) $E_{k+1}^{T}=E_{k}^{T}$ for $\left|T^{C}\right| \leq k$,

(4)

$$
\Sigma_{k+1}^{i}=\Sigma_{k}^{i}-\sum_{\substack{i \in T \\\left|T^{C}\right|=k+1}} E_{k+1}^{T},
$$

(5) $E_{k+1}^{T} \cdot E_{k+1}^{V}=0$ if $T, n+1$ does not $* * V, n+1$,

(6) $\Sigma_{k+1}^{i} \cdot P_{k+1}^{T}=0$ for $i \in T,\left|T^{C}\right| \leq k+1$,

(7) $E_{k+1}^{T} \cdot P_{k+1}^{V}=0$ if $T, n+1$ does not $* * V,\left|V^{C}\right| \leq k+1$,

(8) $E_{k+1}^{T} \cdot \operatorname{ker}\left(\mathbf{A}^{*}\left(B_{1}\right) \rightarrow \mathbf{A}^{*}\left(S_{1}^{T}\right)\right)=0$.

Proof of Claim. The map is induced by sending each of the variables to the corresponding divisor class in $\mathbf{A}^{1}\left(B_{k+1}\right)$ and by the pullback

$$
f_{k+1}^{*}: \mathbf{A}^{*}\left(B_{k}\right) \rightarrow \mathbf{A}^{*}\left(B_{k+1}\right) \text {. }
$$

It is clear from Theorem 1.1, our results on the vital divisors and the preceding lemmas that the map is well defined (i.e. that each of the relations holds). The proposed ring is a quotient of the polynomial ring

$$
\mathbf{A}^{*}\left(B_{k}\right)\left[E_{k+1}^{T}|| T^{C} \mid=k+1\right]
$$

by the quadratic relations. We need only check that the relations described in Lemma 1 are contained in the proposed relations. The relations of Lemma 1 are

and

$$
E_{k+1}^{T} \cdot \operatorname{ker}\left(\mathbf{A}^{*}\left(B_{k}\right) \rightarrow \mathbf{A}^{*}\left(S_{k}^{T}\right)\right) \text { for }\left|T^{C}\right|=k+1
$$

$$
\left(\Sigma_{k}^{i}-E_{k+1}^{T}\right)\left(P_{k}^{T}-E_{k+1}^{T}\right) \quad \text { for }\left|T^{C}\right|=k+1 .
$$

The second can be expressed as

$$
\left(\Sigma_{k+1}^{i}+\sum_{\substack{i \in V \\ T \neq V \\\left|V^{C}\right|=k+1}} E_{k+1}^{V}\right) \cdot P_{k+1}^{T},
$$

which is a sum of the relations of types (6) and (7).

We next show that

is generated by

$$
\operatorname{ker}\left(\mathbf{A}^{*}\left(B_{k}\right) \rightarrow \mathbf{A}^{*}\left(S_{k}^{T}\right)\right)
$$

$$
\operatorname{ker}\left(\mathbf{A}^{*}\left(B_{1}\right) \rightarrow \mathbf{A}^{*}\left(S_{1}^{T}\right)\right)
$$

and

$$
E_{k}^{V}-D_{n}^{V} \quad \text { for } T \subset V,\left|V^{C}\right| \leq k .
$$

By Lemma 3 the second expression is the class of $-P_{k}^{V}$ (since $D_{n}^{V}$ is the class of $P_{1}^{V}$ ) and so this expression (and relation (2)) yields the relation

$$
E_{k+1}^{T} \cdot P_{k+1}^{V} \text { for } T \subset V
$$

which is a relation of type (7). Thus establishing this expression for the kernel finishes the proof of the claim. 
It is enough by induction to show that

$$
\operatorname{ker}\left(\mathbf{A}^{*}\left(B_{k}\right) \rightarrow \mathbf{A}^{*}\left(S_{k}^{T}\right)\right)
$$

is generated by

$$
\operatorname{ker}\left(\mathbf{A}^{*}\left(B_{k-1}\right) \rightarrow \mathbf{A}^{*}\left(S_{k-1}^{T}\right)\right)
$$

and

$$
E_{k}^{V}-D_{n}^{V} \quad \text { for } T \subset V,\left|V^{C}\right|=k \text {. }
$$

This is obtained by the remark immediately following this proof. We choose some ordering on the $S_{k-1}^{V}$ with $\left|V^{C}\right|=k$ (these are the disjoint components of the locus which is blown up by $f_{k}$ ) and make each blowup one at a time. By Lemma 4 the remark may be applied to each of these blowups with $S_{k-1}^{T}$ (or more precisely its strict transform at each stage) playing the role of $W$, the chosen $S_{k-1}^{V}$ playing the role of $X$ and $D_{n}^{V}$ playing the role of $\alpha$.

Remark. Let $\widetilde{Y}$ be the blowup of a scheme $Y$ along a regularly embedded subscheme $X$. Let $W$ be a subscheme of $Y$ such that the intersection $X \cap W$ is a cartier divisor of $W$ (so that $W$ is isomorphic to its strict transform). Assume that the restriction from $\mathbf{A}^{*}(Y)$ to $\mathbf{A}^{*}(X)$ is surjective and that $\alpha \in \mathbf{A}^{1}(Y)$ pulls back to the class of $X \cap W$ in $\mathbf{A}^{1}(W)$. Then

$$
\operatorname{ker} \mathbf{A}^{*}(\widetilde{Y}) \rightarrow \mathbf{A}^{*}(W)
$$

is generated by

$$
\operatorname{ker} \mathbf{A}^{*}(Y) \rightarrow \mathbf{A}^{*}(W)
$$

and $E-\alpha$, (where $E$ is the class of the special divisor).

Proof of Remark. It is clear that each of the given elements is in the kernel of the restriction to $\mathbf{A}^{*}(W)$. Thus we have a map from the quotient of $\mathbf{A}^{*}(\widetilde{Y})$ by these elements to $\mathbf{A}^{*}(W)$ and we need to show that this map is injective. But by Theorem 1 of the appendix this quotient is itself a quotient of $\mathbf{A}^{*}(Y)$ by the kernel of the restriction of $\mathbf{A}^{*}(Y)$ to $\mathbf{A}^{*}(W)$ and the composition is the natural injection. This yields the result.

Repeatedly applying the above result, and using our isomorphism of $\mathbf{X}_{n+1}$ with $B_{n-2}$ we obtain

$$
\mathbf{A}^{*}\left(\mathbf{X}_{n+1}\right)=\frac{\mathbf{A}^{*}\left(B_{1}\right)\left[D_{n+1}^{T, n+1}, D_{n+1}^{T}, D_{n+1}^{i, n+1} \mid T, i \subset\{1,2,3, \ldots, n\}\right]}{\text { the following relations }} .
$$

(1) $D_{n+1}^{T}+D_{n+1}^{T, n+1}=D_{n}^{T}$,

(2) $D_{n+1}^{i, n+1}+\sum_{i \in T} D_{n+1}^{T, n+1}=\Sigma_{1}^{i}$,

(3) $D_{n+1}^{T, n+1} \cdot D_{n+1}^{V, n+1}=0$ if $T, n+1$ does not $* * V, n+1$,

(4) $D_{n+1}^{i, n+1} \cdot D_{n+1}^{T}=0$ for $i \in T$,

(5) $D_{n+1}^{T} \cdot D_{n+1}^{V}=0$ if $T$ does not $* * V$,

(6) $D_{n+1}^{T, n+1} \cdot \operatorname{ker}\left(\mathbf{A}^{*}\left(B_{1}\right) \rightarrow \mathbf{A}^{*}\left(S_{1}^{T}\right)\right)=0$.

(Recall that under the isomorphism of $B_{n-2}$ with $\mathbf{X}_{n+1}, E_{n-2}^{T}, \Sigma_{n-2}^{i}$ and $P_{n-2}^{T}$ correspond to $D_{n+1}^{T, n+1}, D_{n+1}^{i, n+1}$ and $D_{n+1}^{T}$ respectively.)

Since $B_{1}$ is isomorphic to $\mathbf{X}_{n} \times \mathbf{P}^{1}$ we have the expression

$$
\mathbf{A}^{*}\left(B_{1}\right)=\frac{\mathbf{A}^{*}\left(\mathbf{X}_{n}\right)\left[\Sigma_{1}^{i} \mid i \in\{1,2,3, \ldots, n\}\right]}{\left(\Sigma_{1}^{1}\right)^{2}, \Sigma_{1}^{i}=\Pi_{1,2,3, i}+\Sigma_{1}^{1}} .
$$


Here $\Pi_{1,2,3, i}$ denotes the pullback class $\pi_{1,2,3, i}^{*}\left(c_{1}(\mathscr{O}(1))\right)$. Under this isomorphism we have that

$$
\operatorname{ker}\left(\mathbf{A}^{*}\left(B_{1}\right) \rightarrow \mathbf{A}^{*}\left(S_{1}^{T}\right)\right)
$$

is generated by

$$
\operatorname{ker}\left(\mathbf{A}^{*}\left(\mathbf{X}_{n}\right) \rightarrow \mathbf{A}^{*}\left(D_{n}^{T}\right)\right)
$$

and $\Sigma_{1}^{1}-\Pi_{1,2,3, i}$. With this our expression for $\mathbf{A}^{*}\left(\mathbf{X}_{n+1}\right)$ becomes

$$
\mathbf{A}^{*}\left(\mathbf{X}_{n+1}\right)=\frac{\mathbf{A}^{*}\left(\mathbf{X}_{n}\right)\left[D_{n+1}^{V} \mid V \subset\{1,2, \ldots, n+1\}\right]}{\text { the following relations }} .
$$

(1) $D_{n+1}^{T}+D_{n+1}^{T, n+1}=D_{n}^{T}$.

(2) For each $i \in\{1,2,3, \ldots, n\}$

$$
D_{n+1}^{i, n+1}+\sum_{i \in T} D_{n+1}^{T, n+1}=\Pi_{1,2,3, i}+D_{n+1}^{1, n+1}+\sum_{1 \in T} D_{n+1}^{T, n+1} .
$$

$$
\begin{gathered}
\left(D_{n+1}^{1, n+1}+\sum_{1 \in T} D_{n+1}^{T, n+1}\right)^{2} . \\
D_{n+1}^{T, n+1} \cdot \operatorname{ker}\left(\mathbf{A}^{*}\left(\mathbf{X}_{n}\right) \rightarrow \mathbf{A}^{*}\left(D_{n}^{T}\right)\right) . \\
D_{n+1}^{T, n+1} \cdot\left(D^{1, n+1}+\sum_{i \in T} D_{n+1}^{T, n+1}-\Pi_{1,2,3, i}\right) . \\
D_{n+1}^{W} \cdot D_{n+1}^{V} \quad \text { if } W \text { does not } * * V .
\end{gathered}
$$

We perform a few algebraic manipulations to obtain:

Inductive Lemma. $\mathbf{A}^{*}\left(\mathbf{X}_{n+1}\right)$ is a quotient of the polynomial ring over $\mathbf{A}^{*}\left(\mathbf{X}_{n}\right)$ with indeterminants

$$
D_{n+1}^{V} \text { for } V \subset\{1,2, \ldots, n+1\}
$$

defined by the following relations:

I. $D_{n+1}^{T, n+1}+D_{n+1}^{T}=D_{n}^{T}$.

II. For $i, j, k, l$ distinct elements of $\{1,2, \ldots, n+1\}$,

$$
\sum_{\substack{i, j \in W, k, l \notin W \\ \text { or } \\ i, j \notin W, k, l \in W}} D_{n+1}^{W}=\sum_{\substack{i, k \in W, j, l \notin W \\ i, k \notin W, j, l \in W}} D_{n+1}^{W}=\sum_{\substack{i, l \in W, j, k \notin W \\ \text { or } \\ i, l \notin W, j, k \in W}} D_{n+1}^{W} .
$$

III. $D_{n+1}^{W} \cdot D_{n+1}^{V}$ if $W$ does not $* * V$.

Proof. Denote any of the sums in II by $\Pi_{i, j, k, l}$. Observe that by definition it is independent of the ordering of the elements. Its image in $\mathbf{A}^{*}\left(\mathbf{X}_{n+1}\right)$ is the class of the pullback of the canonical linebundle of $\mathbf{P}^{1}$ under the map

$$
\mathbf{X}_{n} \stackrel{\pi_{i, j, k, l}}{\longrightarrow} \mathbf{X}_{4}
$$

by Fact 3 and the remark which immediately follows it. Let $R$ be the proposed ring. We have a surjection of $R$ onto $\mathbf{A}^{*}\left(\mathbf{X}_{n+1}\right)$ and we need only show that 
the relations (2), (3), (4) and (5) of our expression for $\mathbf{A}^{*}\left(\mathbf{X}_{n+1}\right)$ all hold in $R$. Relation (2) can be written as

$$
\sum_{i, n+1 \in T} D_{n+1}^{T}=\Pi_{1,2,3, i}+\Pi_{1,2,3, n+1} .
$$

The left-hand side can be written

$$
\begin{aligned}
\sum_{\substack{1, i, n+1 \in T \\
2,3 \notin T}} D_{n+1}^{T}+\sum_{\substack{2, i, n+1 \in T \\
1,3 \notin T}} D_{n+1}^{T} \\
+\sum_{\substack{2, i, n+1 \in T \\
1,2 \notin T}} D_{n+1}^{T}+\sum_{\substack{i, n+1 \in T \\
1,2,3 \notin T}} D_{n+1}^{T}
\end{aligned}
$$

which in $R$ is equal to

$$
\Pi_{i, n+1,1,3}+\sum_{\substack{1, i, n+1 \in T \\ 2,3 \notin T}} D_{n+1}^{T}+\sum_{\substack{3, i, n+1 \in T \\ 1,2 \notin T}} D_{n+1}^{T} .
$$

The right-hand side can be written as

$$
\begin{aligned}
\sum_{\substack{1, i, n+1 \in T \\
2,3 \notin T}} D_{n+1}^{T}+ & \sum_{\substack{1, i \in T \\
2,3, n+1 \notin T}} D_{n+1}^{T} \\
& +\sum_{\substack{3,2, n+1 \in T \\
1,2 \notin T}} D_{n+1}^{T}+\sum_{\substack{3, n+1 \in T \\
i, 1,2 \notin T}} D_{n+1}^{T}
\end{aligned}
$$

which in $R$ is equal to

$$
\Pi_{1, i, 3, n+1}+\sum_{\substack{1, i, n+1 \in T \\ 2,3 \notin T}} D_{n+1}^{T}+\sum_{\substack{3, i, n+1 \in T \\ 1,2 \notin T}} D_{n+1}^{T} .
$$

Thus we see the left- and right-hand sides are equal in $R$.

In $R$ relation (3) can be written $\Pi_{1,2,3, n+1}^{2}$ which in turn is equal to

$$
\left(\sum_{1, n+1 \in T} D_{n+1}^{T}\right) \cdot\left(\sum_{2, n+1 \varepsilon V} D_{n+1}^{V}\right)
$$

This is a sum of relations of type III. Finally we consider a relation from (4) which can be expressed in $R$ as

$$
D_{n+1}^{T, n+1} \cdot\left(\Pi_{1,2,3, n+1}-\Pi_{1,2,3, i}\right) .
$$

We may assume that 1 is not an element of $T$. We can write this as

$$
D_{n+1}^{T, n+1} \cdot\left(\sum_{\substack{1, n+1 \in V \\ i \notin V}} D_{n+1}^{V}-\sum_{\substack{1, i \in V \\ n+1 \notin V}} D_{n+1}^{V}\right)
$$

which is a sum of relations of type III.

We are now ready to give an expression for $\mathbf{A}^{*}\left(\mathbf{X}_{n}\right)$. We no longer assume that any subset of $\{1,2,3, \ldots, n\}$ considered contains at most one of one two or three. 
Theorem 1.

$$
\mathbf{A}^{*}\left(\mathbf{X}_{n}\right)=\frac{\mathbf{Z}\left[D^{S}|S \subset\{1,2, \ldots, n\}| S|,| S^{C} \mid \geq 2\right]}{\text { the following relations }}
$$

Part I.

(1) $D^{S}=D^{S^{C}}$.

(2) For any four distinct elements $i, j, k, l \in\{1,2, \ldots, n\}$ :

$$
\sum_{\substack{i, j \in S \\ k, l \notin S}} D^{S}=\sum_{\substack{i, k \in S \\ j, l \notin S}} D^{S}=\sum_{\substack{i, l \in S \\ j, k \notin S}} D^{S}
$$

(3) $D^{S} D^{T}$ unless one of the following holds:

$$
S \subset T, \quad T \subset S, \quad S \subset T^{C}, \quad T^{C} \subset S .
$$

Part II.

$$
\operatorname{ker} \mathbf{A}^{*}\left(\mathbf{X}_{n}\right) \rightarrow \mathbf{A}^{*}\left(D_{n}^{T}\right)
$$

is generated by $D^{V}$ unless $V * * T$.

Proof. Notice that all the relations are necessary from the inductive lemma and symmetry.

The proof is by induction. The case of $n=4$ is clear as $\mathbf{X}_{4}=\mathbf{P}^{1}$. We assume the theorem for all $k$ less than $n$ and show that it holds for $n$. By the inductive hypothesis and the inductive lemma (together with some obvious algebra) it follows that Part I holds for $n$. In order to show that Part II holds for $n$ we need to establish the form of

$$
\operatorname{ker} \mathbf{A}^{*}\left(\mathbf{X}_{n}\right) \rightarrow \mathbf{A}^{*}\left(D_{n}^{T}\right)
$$

$D_{n}^{T}$ is isomorphic to the product

$$
\mathbf{X}_{|T|+1} \times \mathbf{X}_{\left|T^{c}\right|+1}
$$

and so (as is established in $\S 2$ ),

$$
\mathbf{A}^{*}\left(D_{n}^{T}\right)=\mathbf{A}^{*}\left(\mathbf{X}_{|T|+1}\right) \otimes \mathbf{A}^{*}\left(\mathbf{X}_{|T|+1}\right)
$$

which by induction is described by Part $\mathrm{I}$. Thus inductively we have explicit expressions for $\mathbf{A}^{*}\left(\mathbf{X}_{n}\right)$ and $\mathbf{A}^{*}\left(D_{n}^{T}\right)$ and need only check that the kernel of the restriction map is generated by the proposed elements.

Since our presentation is symmetric we may assume that $T=\{1,2, \ldots, j\}$ so that

$$
D^{T}=\mathbf{X}_{j+1} \times \mathbf{X}_{n-j+1},
$$

with points $\{1,2,3, \ldots, j, b\}$ on the first factor and $\{b, j+1, j+2, \ldots, n\}$ on the second as described in Fact 2. The vital divisors of $\mathbf{X}_{n}$ pullback to $D^{T}$ as described in Fact 4 . Let $K$ be the ideal generated by the elements given in Part II. It is clear that $K$ is contained in the kernel of the restriction. Let $R=\mathbf{A}^{*}\left(\mathbf{X}_{n}\right) / K$ and let $H$ be the polynomial ring

$$
Z\left[D^{S} \mid S \subset\{1,2,3 \ldots, n\}, S \neq T\right] .
$$

Let $H^{\prime}$ be the polynomial ring $Z\left[D_{j+1}^{V}, D_{n-j+1}^{W}\right]$ where

$$
V \subset\{1,2, \ldots, j+1\}, \quad W \subset\{j, j+1, \ldots, n\} .
$$


It follows from Part I that $H$ maps surjectively onto $R$. (The only divisor which is not present is $D_{n}^{T}$ and this is related to the other variables by relation (2).) Define a map $\pi$ from $H^{\prime}$ to $\mathbf{A}^{*}\left(D^{T}\right)$ as follows:

$$
\begin{aligned}
D_{j+1}^{V, j+1} & \rightarrow D_{j+1}^{V, b} \text { for } V \subset\{1,2, \ldots, j\}, \\
D_{n-j+1}^{W, j} & \rightarrow D_{n-j+1}^{W, b} \text { for } W \subset\{j+1, j+2, \ldots, n\}, \\
D_{j+1}^{V} & \rightarrow D_{j+1}^{V} \text { for } V \subset\{1,2, \ldots, j\}, \\
D_{n-j+1}^{W} & \rightarrow D_{n-j+1}^{W} \text { for } W \subset\{j+1, j+2, \ldots, n\} .
\end{aligned}
$$

This map is a surjection by our expression for $\mathbf{A}^{*}\left(D^{T}\right)$.

Define a map $\phi$ from $H$ to $H^{\prime}$ by

$$
\begin{aligned}
& \phi\left(D_{n}^{S}\right)=0 \quad \text { unless } S * * T \\
& =D_{j+1}^{S} \quad \text { if } S \subset\{1,2, \ldots, j\} \\
& =D_{j+1}^{S \cap\{1,2, \ldots, j+1\}} \text { if } S \supset\{j+1, \ldots, n\} \\
& =D_{n-j+1}^{S} \quad \text { if } S \subset\{j+1, \ldots, n\} \\
& =D_{n-j+1}^{S \cap\{j, j+1, \ldots, n\}} \text { if } S \supset\{1,2, \ldots, j\} \text {. }
\end{aligned}
$$

$\phi$ is clearly surjective.

We have (by Fact 4) a commutative diagram, with all maps surjections

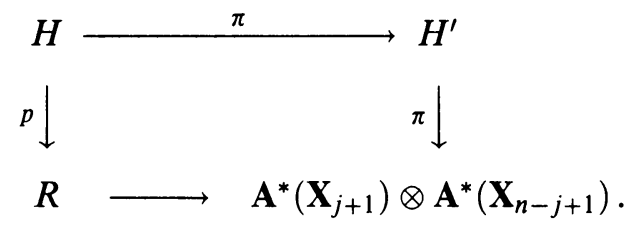

Observe that the kernel of $\phi$ is generated by the $D_{n}^{S}$ where $S$ does not $* * T$. In particular the kernel of $\phi$ is contained in the kernel of $p$ and thus in order to complete the proof it is enough (by any easy diagram chase) to demonstrate that $\operatorname{ker}(\pi) \subset \phi(\operatorname{ker}(p))$. The kernel of $\pi$ is generated by contributions from each factor in $\mathbf{X}_{j+1} \times \mathbf{X}_{n-j+1}$ as described by Part I. We will consider those from the first factor (the second factor being analogous).

A relation of type (1) is of form

$$
D_{j+1}^{S}-D_{j+1}^{S^{C}} \quad \text { for } S, S^{C} \subset\{1,2, \ldots, j+1\} .
$$

We can assume that $j+1$ is not in $S$. Then an inverse image under $\phi$ is $D_{n}^{S}-D_{n}^{S^{C}, j+2, \ldots, n}$ which is in the kernel of $p$.

A typical relation in the kernel of $\pi$ of type (2) is

$$
\sum_{\substack{a, c \in S \\
d, e \notin S \\
s \subset\{1,2, \ldots, j+1\}}} D_{j+1}^{S}-\sum_{\begin{array}{c}
a, d \in S \\
c, e \notin S \\
S \subset\{1,2, \ldots, j+1\}
\end{array}} D_{j+1}^{S} .
$$

Here $a, c, d, e \in\{1,2, \ldots, j+1\}$.

We rewrite this as

$$
\sum_{\substack{a, c, j+1 \in S \\ d, e \notin S}} D_{j+1}^{S}+\sum_{\substack{a, c \in S \\ d, e, j+1 \notin S}} D_{j+1}^{S}-\sum_{\substack{a, d, j+1 \in S \\ c, e \notin S}} D_{j+1}^{S}-\sum_{\substack{a, d \in S \\ c, e, j+1 \notin S}} D_{j+1}^{S} .
$$


An inverse image under $\phi$ of this is

$$
\sum_{\substack{S \supset\{j+1, \ldots, n, a, c\} \\ d, e \notin S}} D_{n}^{S}+\sum_{\substack{a, c \in S \\ d, e \notin S \\ S \subset\{1,2, \ldots, j\}}} D_{n}^{S}-\sum_{\substack{S \supset\{j+1, \ldots, n, a, d\} \\ c, e \notin S}} D_{n}^{S}-\sum_{\substack{a, d \in S \\ c, e \notin S \\ S \subset\{1,2, \ldots, j\}}} D_{n}^{S} \text {. }
$$

We rewrite this last expression as the difference of

$$
\sum_{\substack{a, c \in S \\ d, e \notin S \\ S \subset\{1,2, \ldots, n\}}} D_{n}^{S}-\sum_{\substack{a, d \in S \\ c, e \notin S \\ S \subset\{1,2, \ldots, n\}}} D_{n}^{S}
$$

and

$$
\sum_{\substack{a, c \in S \\
d, e \notin S \\
\text { does not } * * T}} D_{n}^{S}-\sum_{\begin{array}{c}
a, d \in S \\
c, e \notin S \\
S \text { does not } * * T
\end{array}} D_{n}^{S} .
$$

The first term in the sum is an expression of type (2) and so is in the kernel of $p$. The second is a sum of elements in the kernel of $\phi$.

Finally a relation of type (3) in the kernel of $\pi$ is of the form $D_{j+1}^{S} \cdot D_{j+1}^{V}$ where $S, V \subset\{1,2, \ldots, j+1\}$ and $S$ does not $* * V$. Since we have already considered relations of type (1), in order to show that this is in the image of $\operatorname{ker}(\phi)$ we may if necessary replace $S$ by $S^{C}$ and $V$ by $V^{C}$ and so may assume that neither $S$ nor $V$ contains $j+1$. In this case an inverse image under $\phi$ is $D_{n}^{S} \cdot D_{n}^{V}$ which (since $S$ does not $* * V$ ) is in the kernel of $p$.

\section{APPENDIX: REGULAR BLOWUPS}

In this section we relate intersection theoretic properties of $\tilde{Y}$, the blowup of a variety $Y$ along a regularly embedded subvariety $X$, to corresponding properties of $X$ and $Y$.

Specifically, suppose $i: X \hookrightarrow Y$ is a regularly embedded subvariety. Let $\tilde{Y}$ be the blowup of $Y$ along $X$, and let $\widetilde{X}$ be the exceptional divisor. Define $g$ and $j$ by the commutative diagram:

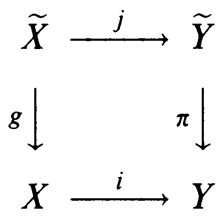

We establish the following results:

Theorem 1. Suppose the map of bivariant rings

$$
i^{*}: \mathbf{A}^{*}(Y) \rightarrow \mathbf{A}^{*}(X)
$$

is surjective, then $\mathbf{A}^{*}(\tilde{Y})$ is isomorphic to

$$
\frac{\mathbf{A}^{*}(Y)[T]}{\left(P(T),\left(T \cdot \operatorname{ker}\left(i^{*}\right)\right)\right.}
$$

where $P(T) \in \mathbf{A}^{*}(Y)[T]$ is any polynomial whose constant term is $[X]$ and whose restriction to $\mathbf{A}^{*}(X)$ is the Chern polynomial of the normal bundle $N_{X} Y$, i.e.

$$
\left(i^{*} P(T)=T^{d}+T^{d-1} c_{1}(N)+\cdots+c_{d-1}(N) T+c_{d}(N)\right.
$$


(where $d=\operatorname{codim}(X, Y)$ ). This isomorphism is induced by

$$
\pi^{*}: \mathbf{A}^{*}(Y) \rightarrow \mathbf{A}^{*}(\tilde{Y})
$$

and by sending $-T$ to the class of the exceptional divisor.

The next result requires a definition.

Definition. A scheme $X$ of characteristic zero is called an HI (for Homology Isomorphism) scheme provided that the canonical map from the Chow groups of $X$ to the homology

$$
\mathbf{A}_{*}(X) \stackrel{\mathrm{cl}}{\longrightarrow} \mathbf{H}_{*}(X)
$$

is an isomorphism.

Observe that if $X$ is an HI scheme then in particular it has no odd homology and hence (by the universal coefficients theorem) its homology (and thus its Chow group) is torsion free.

Theorem 2. If $X$ and $Y$ and HI schemes then so is $\tilde{Y}$.

The theorem in this generality was suggested by Spencer Bloch. For the next theorem assume $X \stackrel{i}{\hookrightarrow} Y$ is of codimension two.

Theorem 3. The map

$$
\mathbf{A}_{k}(Y) \oplus \mathbf{A}_{k-1}(X) \stackrel{\Psi}{\longrightarrow} \mathbf{A}_{k}(\tilde{Y})
$$

defined by

$$
\Psi \stackrel{\text { def }}{=} \pi^{*} \oplus j_{*} g^{*}
$$

is an isomorphism, with inverse $\left(\pi_{*},-g_{*} j^{*}\right)$. Furthermore if $X$ and $Y$ are nonsingular and dual bases exist for their Chow rings, then these bases determine dual bases for $\mathbf{A}^{*}(\widetilde{Y})$ via $\Psi$ as follows:

Let $n$ be the dimensions of $Y$,

$$
\begin{aligned}
\alpha_{1} \in \mathbf{A}^{k}(Y), & \alpha_{2} \in \mathbf{A}^{k-1}(X), \\
\beta_{1} \in \mathbf{A}^{n-k}(Y), & \beta_{2} \in \mathbf{A}^{n-k-1}(X) .
\end{aligned}
$$

Then we have a multiplication table in $\mathbf{A}^{*}(\tilde{Y})$ :

$$
\left\|\begin{array}{ccc}
\cdot & \Psi\left(\alpha_{1}\right) & \Psi\left(\alpha_{2}\right) \\
\Psi\left(\beta_{1}\right) & \alpha_{1} \beta_{1} & 0 \\
\Psi\left(\beta_{2}\right) & 0 & \alpha_{2} \beta_{2}
\end{array}\right\| .
$$

Also if $\gamma \in \mathbf{A}^{n-k}(\widetilde{Y})$, then

$$
\gamma \cdot \Psi\left(\alpha_{1}\right)=\alpha_{1} \cdot \pi_{*}(\gamma) \quad \gamma \cdot \Psi\left(\alpha_{2}\right)=-\alpha_{2} \cdot g_{*} j^{*}(\gamma) .
$$

(This last remark is important since the expression for a cycle as a linear combination of bases elements can be determined by intersecting the cycle with the dual bases in the complimentary dimension.)

\section{Proofs}

Proof of Theorem 1. Let

$$
R=\frac{\mathbf{A}^{*}(Y)[T]}{P(T), T \cdot \operatorname{ker}\left(i^{*}\right)}
$$


and let $f$ be the map

$$
\mathbf{A}^{*}(Y)[T] \stackrel{f}{\longrightarrow} \mathbf{A}^{*}(\tilde{Y})
$$

induced by $\pi^{*}$ and by sending $T$ to $c_{1}(\mathscr{O}(1))$. We show first that $f$ passes to $R$ :

If $c \in \operatorname{ker}\left(i^{*}\right)$ then

$$
\begin{aligned}
f(c \cdot T) & =\pi^{*} c \cdot c_{1}(\mathscr{O}(-1))=-j_{*}\left(j^{*} \pi^{*} c \cdot[j]\right) \\
& =-j_{*}\left(\left(g^{*} i^{*} c\right) \cdot[j]\right)=0 .
\end{aligned}
$$

Define $Q(T)$ by

$$
P(T)=Q(T) \cdot T+[X]
$$

so that

$$
i^{*}(Q)[T]=T^{d-1}+T^{d-2} \cdot c_{1}(N)+\cdots+c_{d-1}(N)
$$

Then

$$
\begin{aligned}
f(Q(T) \cdot T) & =\pi^{*}(Q)\left(c_{1}(\mathscr{O}(1))\right) \cdot c_{1}(\mathscr{O}(1)) \\
& =-j_{*}\left(i^{*} \pi^{*}(Q)\left(c_{1}(\mathscr{O}(1))\right) \cdot[j]\right) \\
& =-j_{*}\left(c_{d-1}\left(g^{*} N / \mathscr{O}(-1)\right) \cdot[j]\right)
\end{aligned}
$$

while

$$
f([X])=\pi^{*}\left(i_{*}[i]\right)=j_{*}\left(g^{*}[i]\right) .
$$

By the excess intersection theorem $[\mathrm{F}]$,

$$
c_{d-1}\left(g^{*} N / \mathscr{O}(-1)\right) \cdot[j]=g^{*}[i]
$$

and thus $f(P(T))=0$ and $f$ factors through $R$. The induced map will also be called $f$. The following sequence is exact

$$
0 \rightarrow \mathbf{A}^{*}(X) \stackrel{\alpha}{\longrightarrow} \mathbf{A}^{*}(\tilde{X}) \oplus \mathbf{A}^{*}(\tilde{Y}) \stackrel{\beta}{\longrightarrow} \mathbf{A}^{*}(\tilde{Y}) \rightarrow 0
$$

where

$$
\begin{gathered}
\alpha(c)=\left(c_{d-1}\left(g^{*} N / \mathscr{O}(-1)\right) \cdot g^{*} c, i_{*}(c \cdot[i])\right), \\
\beta(r, s)=-j_{*}(r \cdot[j])+\pi^{*} s,
\end{gathered}
$$

[F, p. 333]. By assumption (and standard intersection theory of bundles)

$$
\mathbf{A}^{*}(\tilde{X})=\frac{\mathbf{A}^{*}(Y)[T]}{\operatorname{ker}\left(i^{*}\right), P(T)} .
$$

(The isomorphism is induced by sending $T$ to $c_{1}(\mathscr{O}(1))$.)

Multiplication by $T$ induces a map from $\mathbf{A}^{*}(\widetilde{X})$ to $R$ and $\pi^{*}$ induces a map from $\mathbf{A}^{*}(Y)$ to $R$ and together they give a factorization of $\beta$ :

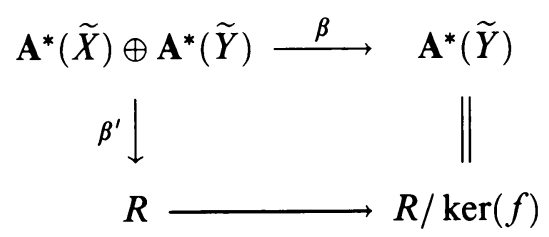


and it is clear from the definitions that $\beta^{\prime}$ is surjective. Thus, in order to show that $f$ is an isomorphism, it suffices to establish that $\beta^{\prime} \circ \alpha=0$. Choose $c \in \mathbf{A}^{*}(X)$, we may assume that $c=i^{*} y$. Then (under the isomorphism $(*)$ )

$$
\begin{aligned}
\beta^{\prime}(\alpha(c)) & =\beta^{\prime}(y \cdot Q(T), y \cdot[X]) \\
& =y \cdot(Q(T) \cdot T+[X]) \\
& =y \cdot(P(T))=0 .
\end{aligned}
$$

This completes the proof.

Proof of Theorem 2. We have a commutative diagram with exact rows

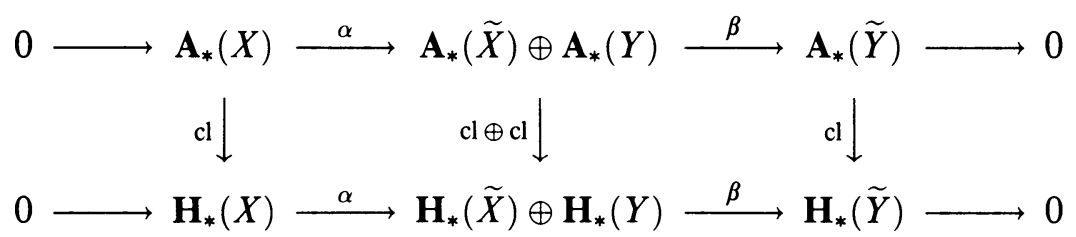

(see for example the proof of Theorem 2.2 in [Kel] from which the result follows.

Proof of Theorem 3. One checks immediately that the given map is a left inverse to $\Psi$. Thus to show $\Psi$ is an isomorphism it suffices to show that $\Psi$ is surjective. From the canonical exact sequence for the Chow groups of a regular blowup (see the proof of the preceding theorem) it suffices to show that $j_{*}(\tilde{x})$ is in the image of $\Psi$ for any $\tilde{x} \in \mathbf{A}_{*}(\widetilde{X})$. $\tilde{x}$ can be written as

$$
g^{*} a+g^{*}(b) \cdot c_{1}\left(g^{*}(N) / \mathscr{O}(-1)\right) .
$$

As $j_{*} g^{*}(a)$ is visibly in the image of $\Psi$ we need only concern ourselves with the second term.

$$
j_{*}\left(g^{*}(b) \cdot c_{1}\left(g^{*}(N) / \mathscr{O}(-1)\right)\right)=\pi^{*}\left(i_{*}(b)\right)
$$

which is in the image of $\Psi$ as required.

The statement regarding dual basis follows from familiar functoriality properties of pushforward and pullback maps and is omitted.

\section{REFERENCES}

[F] W. Fulton, Intersection theory, Springer-Verlag, New York, 1981.

[Kel] S. Keel, Intersection theory of projective linear spaces (preprint).

[Ke2] __ Intersection theory of linear embeddings (preprint).

[Kn] F. Knudsen, Projectivity of the moduli space of stable curves. II, Math. Scand. 52 (1983), $1225-1265$. 